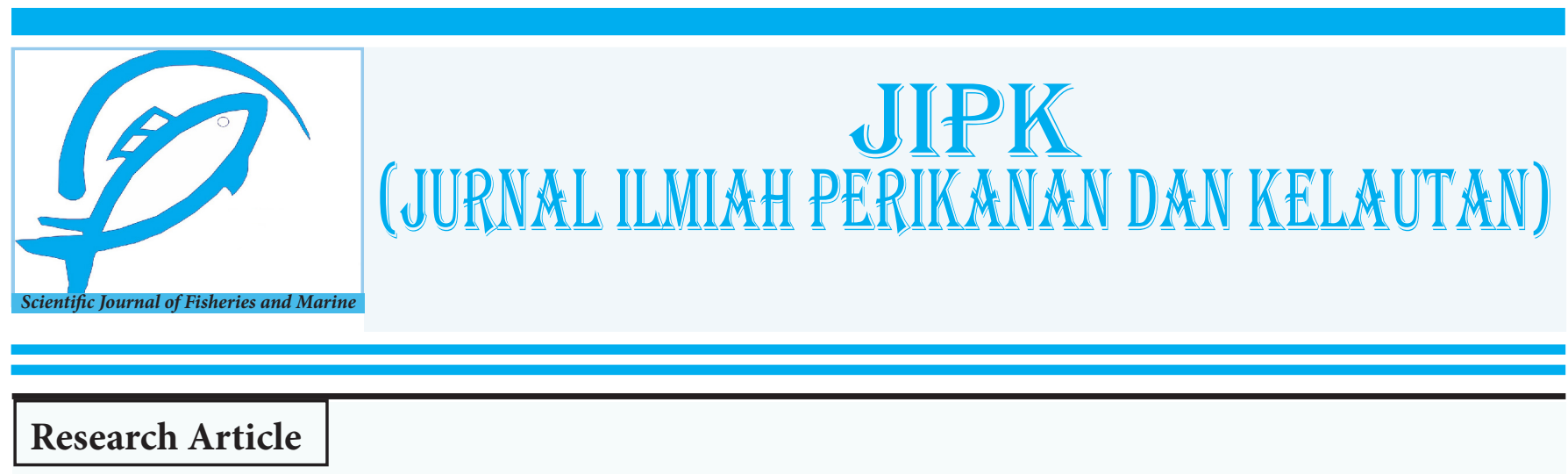

\title{
Marine Biodiversity of Coral Reef Fishes in Pieh Marine Recreational Park After Bleaching and Acanthaster Outbreaks
}

\author{
Risandi Dwirama Putra ${ }^{*}$, (iD Muhammad Abrar², Rikoh Manogar Siringoringo², Ni Wayan \\ Purnamsari², Pradipta Agustina ${ }^{3}$, and Md Jayedul Islam ${ }^{4}$ \\ ${ }^{1}$ Marine Science Department, Faculty of Marine Science and Fisheries, University of Maritime Raja Ali Haji, Tanjungpinang, Indonesia \\ ${ }^{2}$ Reserach Centre of Oceanography, National Research and Innovative Agency (BRIN), Jakarta, 14430. Indonesia \\ ${ }^{3}$ English Language Education Study Program, Faculty of Teacher Training and Education, University of Maritime Raja Ali Haji, Tanjung \\ pinang, 29111. Indonesia \\ ${ }^{4}$ Research Associate Aquatic Bioresource Research Lab, Department of Fisheries Biology and Genetics Sher-e-Bangla Agricultural University, \\ Dhaka. Bangladesh
}

\section{OPEN ACCESS}

\section{$\underline{\text { ARTICLE INFO }}$}

Received: September 21, 2021

Accepted: January 26, 2022

Published: January 26, 2022

*) Corresponding author:

E-mail: risandi@umrah.ac.id

Keywords:

Abundance

Diversity

Index

Massive

Predator

This is an open access article under the CC BY-NC-SA license (https://creativecommons.org/ licenses/by-nc-sa/4.0/)
Abstract

Pieh MRP encountered non-anthropogenic phenomena, precisely massive coral bleaching in 2016, 2017, and massive predators from Acanthaster planci outbreaks in 2018. This study aimed to understand the pattern of coral reef diversity in the core zone and utilization in the MRP area and compare it to non-MRPS locations that accept the same non-anthropogenic pressure conditions. Coral fish sampling using a UVC is categorized into three zones: the core zone, the utilization zone, and outside the MRP area. 8 Families of coral reef fishes were counted based on categories of level function in ecologies and economy. Taxonomic distinctiveness estimates were calculated mathematically for each sample, including species richness and taxonomic diversity were compared among zonation area. Pearson's Coefficient Correlation Matrix was used to measure the correlation relationship between zonation areas. There are 91 species of fish and 3002 individuals found. The richest family in the MRP Core Zone and MRP Utility Zonewas Acanthuridae with 20 species and non-MRP has a lower species richness and abundance of fish communities. The dominant species in Pieh MRP was Ctenochaetus striatus with average abundant per site $(21.3 \pm 7.62, \mathrm{n}=3)$. Acanthuridae represents $55.98 \%$ of the total biomass in MRP-Core Zone, $63.13 \%$ in MRP-Utility Zone, and $41.55 \%$ in Non-MRP Area. This study showed the number of species and populations from corallivores fishes have decreased but has been an increase in herbivorous and carnivore diversity. The diversity indices (H') and ENS also shows no differ significantly between zonation.

Cite this as: Putra, R. D., Abrar, M., Siringoringo, R. M., Purnamsari, N. W., Agustina, P. \& Islam, M. J. (2022). Marine Biodiversity of Coral Reef Fishes in Pieh Marine Recreational Park After Bleaching and Acanthaster Outbreaks. Jurnal Ilmiah Perikanan dan Kelautan, 14(1):48-70. http://doi.org/10.20473/jipk.v14i1.30133 


\section{Introduction}

Awareness of the threat of coral reef ecosystems has increased globally (Gardner et al., 2003). Continuous pressure on coral reefs ecosystems caused by anthropogenic and non-anthropogenic factors leads to a decline in coral cover globally and reduce ecological conditions in the coastal ecosystem (Harvey et al., 2018; Heery et al., 2018; Hoegh-Guldberg et al., 2017). The decline of the coral reefs ecosystem is a major concern for ecologists. The pressure from non-anthropogenic and anthropogenic factors including global warming with related coral bleaching (Jones et al., 2004), increasing human populations and activities in coastal areas (Trenouth and Campbel1, 2013), overfishing (McClanahan and Jadot, 2017), and destructive fishing activities (McManus and Polsenberg, 2004) can cause loss of flora and fauna species that depend on this ecosystem. The ecological role of various species of flora and fauna is very crucial for the functioning of coral reef ecosystems. The healthy functioning of coral reef ecosystem services is highly beneficial to human society (Thibaut and Connolly, 2013).

The usefulness and high ecological function of coral reef ecosystems increase awareness about the necessity to manage and reduce pressure on coral reef ecosystems, including by decreasing anthropogenic stressor caused by increasing population and human activities (Bruno and Valdivia, 2016; Trenouth and Campbell, 2013) with Marine Protection Area management. The formation of Marine Protects Area (MPA) and Marine Recreational Park (MRP) is very appropriate in succeeding in the controlled pressure on coral reef ecosystems. As in Pieh Island and the surrounding sea located in West Sumatra that used as the location of MRPs. Formation Pieh as MRPs is formed by legislation in the Minister of Forestry Decree Number 070 / Kpts-II / 2000, dated March 18, 2000, as Nature Marine Tourism Park (NMTP) (Abrar et al., 2014), In its development, the implementation of Pieh Island as NMTP management submitted to the Ministry of Maritime Affairs and Fisheries under the Decree of the Ministry of Maritime Affairs and Fisheries Number Kep. 70 / Men / 2009 and became the Marine Recreational Park (MRP) of Pieh Island and the Surrounding Sea. Currently, Pieh MRP is managed by the Directorate General of Treasury Affairs Pekanbaru with field technical implementation work units stationed in Padang City, West Sumatra Province (Abrar et al., 2014). The conservation area program in MRPs in Pieh has implemented specific zoning areas and divided into two main zones namely core zone (no-take zone) and the utilization zone. The core zone in Pieh MRP is part of the protected area of small islands, which is intended for the protection of habitats and populations, the resources and their use is limited to research, while the utilization zone in Pieh MRP is primarily utilized for the benefit of nature tourism and other environmental conditions/services. This division of zones provides an appropriate level of protection for a variety of representative species and habitats (Hammerton, 2017; Himes, 2007) and provides opportunities for the government and the community to manage their marine areas.

In the past few decades, global efforts have been made to develop marine protected areas as both MPAs and MRPs. This program is not only arranged in Indonesia; but also in global effort to preserve increasingly threatened marine ecosystems to protect genetic diversity, species ecosystems extensively (Abelson et al. 2016; Hammerton, 2017). Although there are several regional and global successes in establishing MPAs and MRPs, the fact is that coral reefs around the world is experiencing a severe decline (Attamimi, 2019; Bellwood et al., 2004; Paddack et al., 2009). The weak management and supervision have resulted in the objectives-to manage coral reef ecosystems not functioning correctly. Some problems are excessive utilization and harvesting (Jackson et al., 2001; Pandolfi et al., 2003), pollution (McCulloch et al., 2003), disease (Harvell et al., 2002), and non-anthropogenic factors such as climate change (Hughes et al., 2003; Wilkinson, 2008). At present, the remaining coral populations are increasingly affected by increasingly prevalent coral diseases and climate change that triggered coral bleaching (Gardner et al., 2003; Putra et al., 2019) and marine predator outbreaks (A. planci) (Kayal et al., 2012; Leray et al., 2012; Mellin et al., 2016). This degradation of coral reef cover health impacts the existence of coral reefs in the long term and will indirectly affect several related biotas that depend on coral reefs, especially reef fish. A decrease in the structural complexity of reef habitats is often associated with changes in fish communities and influence on the role of fish in coral reefs (Woodhead et al., 2019). Reef fish also have an essential role in the recovery of coral reefs and the presence of certain species can accelerate the recovery of coral reefs (Cheal et al., 2008)

In its development, the Pieh MRP encountered non-anthropogenic phenomena, in particular massive coral bleaching in 2016, 2017, and massive predators from crown-of-thorns starfish $(A$. planci) outbreaks in 2018. The pressure caused almost the majority of Pieh MRP areas to change in coral reef ecosystems. The emergence of Rubble and Dead Coral Algae (DCA) after disturbance of non-anthropogenic pressure in the MRP in Pieh increases deterioration of coral reef ecosystems. The A. planci outbreaks in Pieh MRP in 2018 contributed to the decrease of health of the coral reef cover and reduced coral reefs recovery. The Directorate General of Treasury, the Ministry of Maritime Affairs 
and Fisheries of Indonesia with several NGOs have tried to maintain and control the location by removing nearly 800 individuals of $A$. planci in the coral reef to maintain and succeed in the MPA management program. The increasing of non-anthropogenic stressor as massive coral bleaching and the appearance of marine predators $(A$. planci) are the main concerns in evaluating the health of coral reefs globally because these cause a shift from living corals to algal dominance (McManus and Polsenberg, 2004). The dominance of algal growth causes disruption on hard coral, where macro and fleshy algae covers live coral tissue and cause disturbance to coral growth (Chabanet et al., 2016). The response of coral reef fish to coral ecosystems after the impact of these pressures can be an essential factor influencing the success of this protected area (De Freitas et al., 2013). It is necessary to understand the pattern of coral reef diversity and to recognize the influence on reef fish diversity in order to support coral reef recovery from the magnitude of non-anthropogenic pressure and to assist in making policies and managing MRPs sustainability. It is necessary to understand the influence and role of the core zones and utilization zones for changing the diversity of reef fish after the bleaching and Acanthaster outbreak in Pieh MRP. Understanding the patterns of reef fish diversity can help support coral reef recovery from the magnitude of non-anthropogenic pressure and can be used to make policies and management for sustainable MRPs. This study provided the biodiversity of reef fish after the phenomenon of coral bleaching and $A$. planci outbreaks in the Pieh MRP, based on different zonation: Core Zone (No-take zone), Utility Zone (Recreational Zone) and we compare with Non-MPA (Fishing Zone) areas. The phenomenon of coral bleaching and the $A$. planci outbreaks impact on coral conditions and lead to the dominance of algal growth in the Pieh areas. This study aims to examine the condition of coral ecosystems by evaluating coral reef fish composition after the appearance of bleaching phenomena and outbreaks of A. planci to found a possible recovery of the coral ecosystem at MRPs Pieh location.

\section{Materials and Methods}

\subsection{Study Sites}

The study was conducted in March 2019 in Marine Recreational Park (MRP) in Pieh and the surrounding sea, consisting of 5 small uninhabited islands (Pieh Island, Air Island, Bando Island, Pandan Island, and Toran Island) (Figure 1). Marine Recreational Park (MRP) Pieh with 39,900 ha covers a strip along western coastline, western of Padang, Indonesia. The coral reef ecosystem spread out, extending north-south along the shoreline of West Sumatra, and the main benthic features were fringing reef with Pocilloporiid, and
Acroporiid (Abrar et al., 2014). Management and utilization of (MRP) Pieh area focused on environmentally friendly marine tourism by taking into account the aspects of shared ownership, management, and responsibility, to get harmonized with its use as marine tourism. These areas were also a fishing location (fishing ground) for local fishermen, as evidenced by the number of Fish Aggregating Devices (FADs) in the sustainable utilization zone. Almost all islands become dive sites with clear waters, and beautiful coral reefs as the main attraction. Conservation directed towards efforts to protect the diversity of marine biota in this area, especially rare and protected biota, several marine mammals such as dolphins and whales, and near-threatened fish species including sharks and Napoleon (Abrar et al., 2014). The sampling design of the research location was categorized into three group zonation areas: (1) core zone area (no-take zone) in MRP, (2) utilization zone area (recreational zone) in MRP, and (3) outside MRP area (fishing zone). A total of thirteen sampling sites (10 sites within the MRP area, divided by 2 categorized zonation that was five sites in core zone area in MRP and five sites in utilization zone area in MRP), other three sites outside MRP area selected to compare coral reef fishes diversity, composition, and biomass. The geographical positioning system (GPS) locations of the sites was recorded (Table 1)

\subsection{Data Collection}

The coral reef fishes were initially surveyed using Underwater Visual Censuses (UVC) (Floeter et al., 2004). Underwater Visual Census method (UVC) for reef fishes was done along $70 \mathrm{~m}$ of transect line straight and follows the contour of the shore and laid out parallel to the shore (Bouchon-Navaro, 1981; Giyanto et al., 2014). The basic unit of data collection recording fish encountered within 70 meters and 2.5 meters of observation on either side to record fish species, estimated length, abundance, and several reef characteristics of interest (e.g., live coral cover, algal cover, depth, visibility, etc.) (Zenone et al., 2017). This observation generated an observation area of $350 \mathrm{~m}^{2}$ (Putra et al., 2018). All observations of UVC made while scuba diving at depths of 4-10 $\mathrm{m}$ in the coral reef ecosystem. Constant speed was maintained as far as possible to prevent the recounting of fish (Buxton and Smale, 1989). There were 8 families of coral reef fishes that were counted based on its ecologies and economical function. The first category was a coral feeder or corralivore (Chaetodontidae), the second category in herbivore fishes group (Acanthuridae, Scaridae, and Siganidae), the third category is carnivore fishes group (Haemulidae, Lethrinidae, Lutjanidae and Serranidae). The corralivore fish (Chaetodontidae) are indicator fishes that are strongly associated with coral reef health and used to 
Putra et al. / JIPK, 14(1):48-70
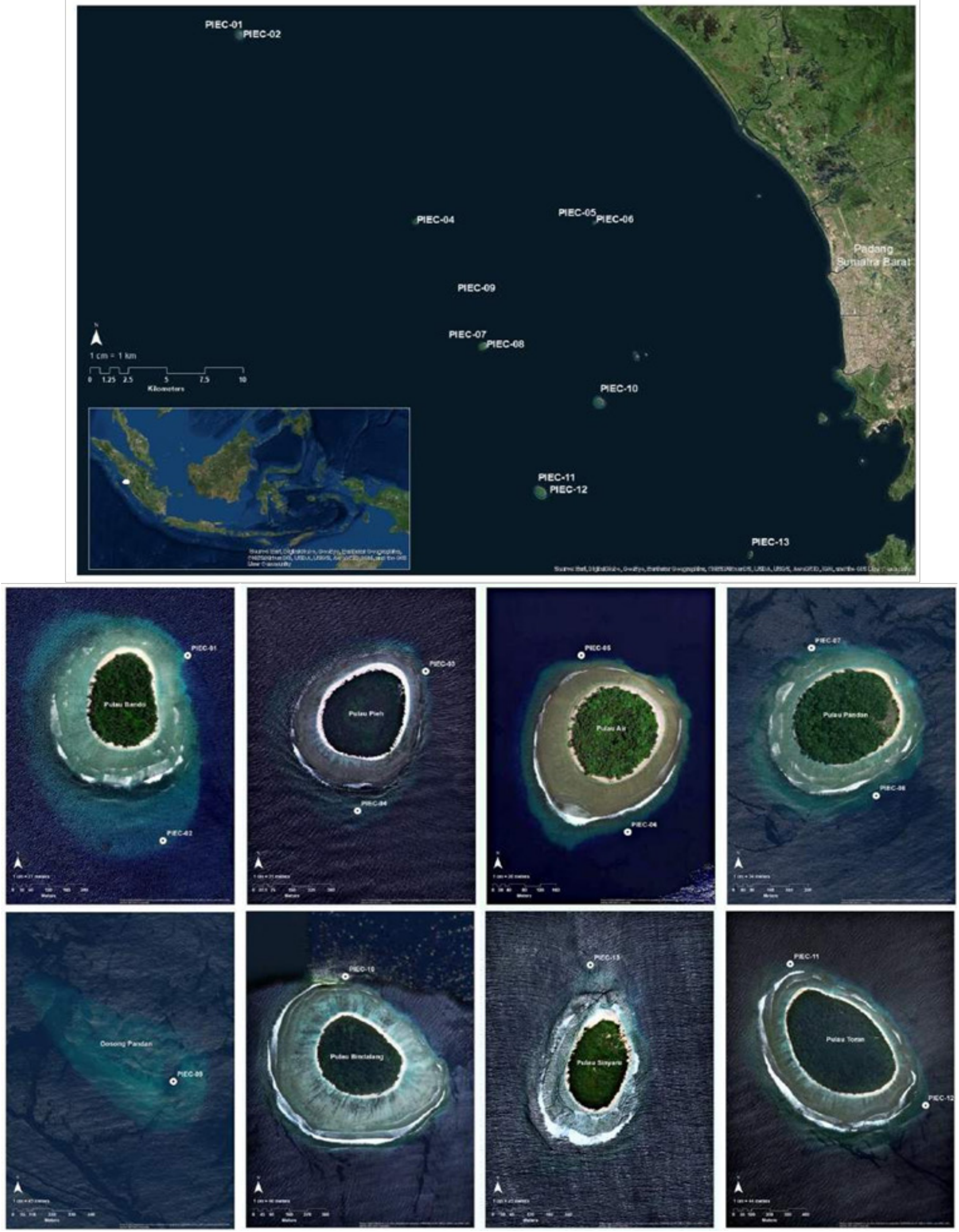

Figure 1. Map of study sites: MRP Area (Air Island, Bando Island, Pandan Island, Pieh Island, and Toran Island) and the Non-MRP Area (Bindalang Island, Sinyaru Island, and Gosong Pandan Reef) Padang City, West Sumatra 
JIPK. Volume 14 No 1. April 2022 / Marine Biodiversity of Coral Reef Fishes in Pieh Marine Recreational Park....

determine the coral reef health. The majority of herbivore and carnivore fish in these family group are economical target fish were fish caught by fishermen because of their high economic, commercial value and usually sold on the market for food consumption (Corrales et al., 2015; Madduppa et al., 2014).

\subsection{Data Analysis}

The number of total individual in each species, estimates of reef fish total length recorded, and bias measurement of estimation total length for each individual reef fishes should not exceed $5 \mathrm{~cm}$ (Buxton and Smale, 1989). The estimated length of fish data was converted to fish biomass using length-weight relationships for fish species using the following equation $\mathrm{W}=\mathrm{a} \times \mathrm{L}^{\mathrm{b}}$, where $\mathrm{W}$ is weight $(\mathrm{g}), \mathrm{L}$ is total length $(\mathrm{cm})$, and parameters $\mathrm{a}$ and $\mathrm{b}$ are constants for the allometric growth equation (Luiz et al., 2015). These coefficients come from data published in FishBase (fishbase.org) when coefficient values not found for the species; this study used coefficients defined for a species with morphological characteristics similar to the observed species. The total biomass for each station corresponds to the total weight of all fish per unit area $\left(\mathrm{kg} / \mathrm{m}^{2}\right)$. The identification of reef fish is based primarily on the identification of reef fish by (Allen et al., 2003). Most values and attributes were obtained from FishBase (Froese and Pauly, 2017). Reef fish data collected were analyzed for ranking diversity based on family per zonation (MRPCZ, MRP-UZ, and Non-MRP) and for each species of fish will be analyzed based on rank species per zonation.
In the particular case of zonation area of MRP, several taxonomic distinctiveness estimates were calculated mathematically for each sample, including species richness (the number of fish taxa per area) and taxonomic diversity by the Shannon- Wiener diversity index $\left(H^{\prime}\right)$, Alpha fisher Index $(A F)$, ENS (Effective Number of Species) and Simpson Dominance Index $(S)$ were compared among zonation area. To measure how strong the correlation of relationships between zonation area in each site using Pearson's coefficient correlation matrix (CCM). This study compared the changing coral reef composition (species richness, abundance and biomass) before (2014) and after (2019) bleaching phenomena and $A$. planci outbreaks.

\section{Results and Discussion}

\subsection{Result}

A total of 91 fish species and 3002 individuals are observed from the eight-coral reef fish families (Chaetodontidae, Acanthuridae, Scaridae, Siganidae, Haemulidae, Lethrinidae, Lutjanidae, and Serranidae) in 13 sites location. The most diverse family in core zone Area MRP (MRP-CZ) is Acanthuridae with 20 species, followed by Chaetodontidae with 18 species, Scaridae with 13 species, Serranidae with eight species, Lutjanidae with six species, Siganidae with three species, Haemulidae with three species, and Lethrinidae with three species (Table 2). Fish communities in core zone area MRP are dominated by Acanthuridae, which represented $60.73 \%$ of the total number of individuals coral

Table 1. Location coordinates of sites and zonation in Marine Recreational Park Pieh

\begin{tabular}{|c|c|c|c|c|c|c|}
\hline No & Location & Sites.id & Zonation & $\begin{array}{c}\begin{array}{c}\text { Depth } \\
\text { (meters) }\end{array} \\
\end{array}$ & Longitude & Latitude \\
\hline 1 & Bando Island & PIEC.01 & MRP-CZ & 7 & $99^{\circ} 59^{\prime} 52.738^{\prime \prime} \mathrm{E}$ & $0^{\circ} 45^{\prime} 53.712^{\prime \prime} \mathrm{S}$ \\
\hline 2 & Pandan Island & PIEC.02 & MRP-UZ & 9 & $99^{\circ} 59^{\prime} 57.012 ” \mathrm{E}$ & $0^{\circ} 45^{\prime} 41.508 " \mathrm{~S}$ \\
\hline 3 & Pieh Island & PIEC.03 & MRP-UZ & 7 & $100^{\circ} 06^{\prime} 9.360 ” \mathrm{E}$ & $0^{\circ} 52^{\prime} 28.128^{\prime \prime} \mathrm{S}$ \\
\hline 4 & Pieh Island & PIEC.04 & MRP-CZ & 5 & $100^{\circ} 06^{\prime} 0.360^{\prime \prime} \mathrm{E}$ & $0^{\circ} 522^{\prime} 38.712 " \mathrm{~S}$ \\
\hline 5 & Air island & PIEC.05 & MRP-UZ & 5 & $100^{\circ} 12^{\prime} 25.56^{\prime \prime} \mathrm{E}$ & $0^{\circ} 52^{\prime} 28.092 ” \mathrm{~S}$ \\
\hline 6 & Air Island & PIEC.06 & MRP-CZ & 6 & $100^{\circ} 12^{\prime} 19.80 ” \mathrm{E}$ & $0^{\circ} 52^{\prime} 37.632^{\prime \prime} \mathrm{S}$ \\
\hline 7 & Pandan Island & PIEC.07 & MRP-UZ & 10 & $100^{\circ} 08^{\prime} 34.08^{\prime \prime} \mathrm{E}$ & $0^{\circ} 56^{\prime} 55.788^{\prime \prime} \mathrm{S}$ \\
\hline 8 & Pandan Island & PIEC.08 & MRP-CZ & 5 & $100^{\circ} 08^{\prime} 27.96 ” \mathrm{E}$ & $0^{\circ} 57^{\prime} 09.468 " \mathrm{~S}$ \\
\hline 9 & Gosong Pandan Island & PIEC.09 & Non-MRP & 8 & $100^{\circ} 07^{\prime} 26.76^{\prime \prime} \mathrm{E}$ & $0^{\circ} 55^{\prime} 6.8988 ” \mathrm{~S}$ \\
\hline 10 & Bindalang Island & PIEC.10 & Non-MRP & 5 & $100^{\circ} 12^{\prime} 28.44^{\prime \prime} \mathrm{E}$ & $0^{\circ} 58^{\prime} 46.499 \prime \mathrm{S}$ \\
\hline 11 & Toran Island & PIEC.11 & MRP-CZ & 6 & $100^{\circ} 10^{\prime} 16.68^{\prime \prime} \mathrm{E}$ & $1^{\circ} 02^{\prime} 0.8880 " \mathrm{~S}$ \\
\hline 12 & Toran Island & PIEC. 12 & MRP-UZ & 7 & $100^{\circ} 10^{\prime} 37.56^{\prime \prime} \mathrm{E}$ & $1^{\circ} 02,15.360 " \mathrm{~S}$ \\
\hline 13 & Sinyaru Island & PIEC.13 & Non-MRP & 4 & $100^{\circ} 17^{\prime} 48.84$ ' $\mathrm{E}$ & $1^{\circ} 04^{\prime} 21.900 " \mathrm{~S}$ \\
\hline
\end{tabular}

Note: MRP CZ (Marine Recreational Park - Core Zone); MRP-UZ (Marine Recreational Park-Utility Zone); NonMRP (non-Marine Recreational Park). 
reef fishes with Ctenochaetus striatus $(48.4 \pm 10.97, \mathrm{n}$ $=5)$ and Acanthurus lineatus $(27.4 \pm 9.48, \mathrm{n}=5)$ being the two most average abundant species per site (Table 2). The most diverse family of coral reef fishes in utilization zone area MRP (MRP-UZ) almost similar condition in MRP-CZ, where Acanthuridae is richest family with 17 species, followed by Chaetodontidae with 15 species, Serranidae with 11 species, Scaridae with ten species, Siganidae with five species, Lutjanidae with five species, Lethrinidae with three species, and Haemulidae with two species (Figure 2). Fish communities in MRP-UZ are dominated by Acanthuridae, which represent $61.13 \%$ of the total number of individuals coral reef fishes with $C$. striatus $(31.8 \pm 5.76, \mathrm{n}=$ $5)$ and $A$. lineatus $(27.0 \pm 7.11, \mathrm{n}=5)$ being the two most average abundant per site (Table 2).

Outside Area MRP (Non-MRP) has a lower species richness and fish community abundance, where Acanthuridae is richest family with 17 species, followed by Chaetodontidae with 16 species, Scaridae with nine species, Serranidae with six species, Siganidae with five species, Lutjanidae with 5 species, Haemulidae with 1 species, and Lethrinidae with one species (Figure 2). Fish communities in non-MRP were dominated by Acanthuridae, which represented $47.74 \%$ of the total number of individuals coral reef fishes with $C$. striatus $(21.3 \pm 7.62, \mathrm{n}=3)$ being the most average abundant per site and followed by Acanthurus tristis (14.3 $\pm 8.69, \mathrm{n}=3)$, Forcipiger flavissimus $(10.3 \pm 3.53, \mathrm{n}=3)$, Siganus virgatus $(10.3 \pm 2.96, \mathrm{n}=3)$, A. lineatus $(10.0 \pm$ $3.46, \mathrm{n}=3)$, Zebrasoma scopas $(9.3 \pm 4.81, \mathrm{n}=3)$, Chlorurus capistratoides $(8.3 \pm 4.48, \mathrm{n}=3)$, Cephalopolis argus $(8.3 \pm 4.48, \mathrm{n}=3)$, Heniochus pleurotaenia $(7.7 \pm 1.76$, $\mathrm{n}=3)$, and Naso hexacanthus $(7.7 \pm 5.78, \mathrm{n}=3)$ (Table 2). Through the Kruskal-Wallis test to species richness, mean abundance and mean biomass of composition reef fishes in three region area (core zone, utility zone, and non-MRP), the significant difference was showed by species richness (Kruskal-Wallis chi-squared $=12.558, \mathrm{df}=2, \mathrm{p}$-value $=0.001876)($ Figure 2$)$. Pairwise Wilcoxon test for the species diversity of reef fish shows a significant difference between MRP-CZ with Non-MRP (p-value $=0.0220$ ) and between MRP-UZ with Non-MRP ( $p$-value $=0.0015$ ). The mean abundance and biomass of reef fish shows no differences between 3 locations with (Kruskal-Wallis chi-squared $=2.66, \mathrm{df}=2, \mathrm{p}$-value $=0.2645)$ and (Kruskal-Wallis chi-squared $=1.0339, \mathrm{df}=2, \mathrm{p}$-value $=0.5963)$, respectively (Figure 2).

Table 2. List of taxa, number of individuals ( $( \pm \mathrm{SE}$ individual/350 m2), in Marine Recreational Park (Core Zone and Utilization Zone) and Non-Marine Recreational Park in Pieh after non-anthropogenic pressure condition (2019)

\begin{tabular}{|c|c|c|c|c|c|}
\hline Species & Common Names & $\begin{array}{l}\text { Trophic } \\
\text { Status }\end{array}$ & $\begin{array}{c}\text { CZ-MRP } \\
\mathbf{n}=5\end{array}$ & UZ-MRP n=5 & $\begin{array}{c}\text { (N-MRP) } \\
\quad n=3\end{array}$ \\
\hline \multicolumn{6}{|l|}{ Chaetodontidae } \\
\hline Chaetodon citrinellus (Cuvier, 1831) & Speckled butterflyfish & Corallivores & $2.2 \pm 1.28$ & $2.6 \pm 1.08$ & $3.3 \pm 3.33$ \\
\hline Chaetodon collare (Bloch, 1787) & Redtail butterflyfish & Corallivores & $0.2 \pm 0.20$ & $1.6 \pm 0.98$ & $3.3 \pm 2.03$ \\
\hline Chaetodon falcula (Bloch, 1795) & Blackwedged butterflyfish & Corallivores & $1.6 \pm 0.75$ & $1.0 \pm 0.63$ & $0.3 \pm 0.33$ \\
\hline Chaetodon kleinii (Bloch, 1790) & Sunburst butterflyfish & Corallivores & $2.4 \pm 0.93$ & - & $1.0 \pm 0.58$ \\
\hline Chaetodon lunula (Lacepède, 1802) & Raccoon butterflyfish & Corallivores & $0.4 \pm 0.40$ & $0.2 \pm 0.20$ & $0.7 \pm 0.67$ \\
\hline Chaetodon meyeri (Bloch \& Schneider, 1801) & Scrawled butterflyfish & Corallivores & $0.6 \pm 0.24$ & $0.6 \pm 0.60$ & $1.0 \pm 1.00$ \\
\hline Chaetodon ornatissimus (Cuvier, 1831) & Ornate butterflyfish & Corallivores & $1.0 \pm 0.63$ & $0.8 \pm 0.49$ & $0.3 \pm 0.33$ \\
\hline Chaetodon oxycephalus (Bleeker, 1853) & Spot-nape butterflyfish & Corallivores & $0.6 \pm 0.40$ & - & - \\
\hline Chaetodon rafflesi (Bennett, 1830) & Latticed butterflyfish & Corallivores & $1.6 \pm 0.93$ & $1.6 \pm 0.75$ & $2.0 \pm 1.15$ \\
\hline Chaetodon triangulum (Cuvier, 1831) & Triangle butterflyfish & Corallivores & $1.4 \pm 0.87$ & $1.0 \pm 0.55$ & $0.3 \pm 0.33$ \\
\hline $\begin{array}{l}\text { Chaetodon trifascialis (Quoy \& Gaimard, } \\
1825 \text { ) }\end{array}$ & Chevron butterflyfish & Corallivores & $0.2 \pm 0.20$ & $0.4 \pm 0.40$ & $0.3 \pm 0.33$ \\
\hline Chaetodon trifasciatus (Park, 1797) & Melon butterflyfish & Corallivores & $4.0 \pm 1.41$ & $5.8 \pm 1.28$ & $4.3 \pm 1.45$ \\
\hline Chaetodon vagabundus (Linnaeus, 1758) & Vagabond butterflyfish & Corallivores & $4.0 \pm 1.30$ & $4.8 \pm 1.71$ & $5.7 \pm 3.18$ \\
\hline $\begin{array}{l}\text { Forcipiger flavissimus (Jordan \& McGregor, } \\
\text { 1898) }\end{array}$ & Longnose butterfly fish & Corallivores & $5.2 \pm 2.22$ & $5.6 \pm 1.08$ & $10.3 \pm 3.53$ \\
\hline Forcipiger longirostris (Broussonet, 1782) & Longnose butterflyfish & Corallivores & - & $0.4 \pm 0.40$ & - \\
\hline Hemitaurichthys zoster (Bennett, 1831) & $\begin{array}{l}\text { Brown-and-white butter- } \\
\text { flyfish }\end{array}$ & Corallivores & $0.4 \pm 0.24$ & - & - \\
\hline Heniochus acuminatus (Linnaeus, 1758) & Pennant coralfish & Corallivores & $0.2 \pm 0.20$ & - & $0.7 \pm 0.67$ \\
\hline
\end{tabular}


JIPK. Volume 14 No 1. April 2022 / Marine Biodiversity of Coral Reef Fishes in Pieh Marine Recreational Park....

Heniochus pleurotaenia (Ahl, 1923)

Heniochus singularius (Smith \& Radcliffe, 1911)

\section{Acanthuridae}

Acanthurus nubilus (Fowler \& Bean, 1929)

Acanthurus grammoptilus (Richardson, 1843)

Acanthurus leucocheilus (Herre, 1927)

Acanthurus leucosternon (Bennett, 1833)

Acanthurus lineatus (Linnaeus, 1758)

Acanthurus maculiceps (Ahl, 1923)

Acanthurus mata (Cuvier, 1829)

Acanthurus nigricans (Linnaeus, 1758)

Acanthurus thompsoni (Fowler, 1923)

Acanthurus triostegus (Linnaeus, 1758)

Acanthurus tristis (Randall, 1993)

Acanthurus tennentii (Günther, 1861)

Ctenochaetus binotatus (Randall, 1955)

Ctenochaetus striatus (Quoy \& Gaimard, 1825)

Ctenochaetus truncatus (Randall \& Clements, 2001)

Naso brachycentron (Valenciennes, 1835)

Naso hexacanthus (Bleeker, 1855)

Naso elegans (Rüppell, 1829)

Naso lituratus (Forster, 1801)

Naso thynnoides (Cuvier, 1829)

Naso unicornis (Forsskål, 1775)

Naso vlamingii (Valenciennes, 1835)

Zebrasoma scopas (Cuvier, 1829)

Zebrasoma veliferum (Bloch, 1795)

\section{Scaridae}

Cetoscarus bicolor (Rüppell, 1829)

Chlorurus bleekeri (de Beaufort, 1940)

Chlorurus capistratoides (Bleeker, 1847)

Chlorurus sordidus (Forsskål, 1775)

Chlorurus troschelii (Bleeker, 1853)

Scarus dimidiatus (Bleeker, 1859)

Scarus ghobban (Forsskål, 1775)

Scarus niger (Forsskål, 1775)

Scarus oviceps (Valenciennes, 1840

Scarus prasiognathos (Valenciennes, 1840)

Scarus quoyi (Valenciennes, 1840

Scarus rubroviolaceus (Bleeker, 1847)

Scarus tricolor (Bleeker, 1847

Scarus viridifucatus (Smith, 1956)

Siganidae
Phantom bannerfish

Singular bannerfish

Bluelined surgeon

Finelined surgeonfish

Palelipped surgeonfish

Powderblue surgeonfish

Lined surgeonfish

White-freckled surgeonfish

Elongate surgeonfish

Whitecheek surgeonfish

Thompson's surgeonfish

Convict surgeonfish

Indian Ocean mimic surgeonfish

Doubleband surgeonfish

Twospot surgeonfish

Striated surgeonfish

Indian gold-ring bristle-tooth

Humpback unicornfish

Sleek unicornfish

Elegant unicornfish

Orangespine unicornfish

Oneknife unicornfish

Bluespine unicornfish

Bignose unicornfish

Twotone tang

Sailfin tang

Bicolour parrotfish

Bleeker's parrotfish

Indian parrotfish

Daisy parrotfish

Troschel's parrotfish

Yellowbarred parrotfish

Blue-barred parrotfish

Dusky parrotfish

Dark capped parrotfish

Singapore parrotfish

Quoy's parrotfish

Ember parrotfish

Tricolour parrotfish

Roundhead parrotfish
Corallivores $\quad 7.0 \pm 1.92 \quad 7.6 \pm 2.54 \quad 7.7 \pm 1.76$

$\begin{array}{llll}\text { Corallivores } & 4.8 \pm 0.92 & 4.2 \pm 0.86 & 2.0 \pm 0.58\end{array}$

Herbivores

$5.2 \pm 3.07$

$0.6 \pm 0.60$

Herbivores

Herbivores

$0.2 \pm 0.20$

$10.4 \pm$

9.67

$27.4 \pm$

9.48

$1.4 \pm 1.17$

$0.2 \pm 0.20$

Herbivores

Herbivores

$0.6 \pm 0.40$

$3.0 \pm 2.07$

$1.0 \pm 1.00$

Herbivores

$5.0 \pm 1.38$

$13.0 \pm 3.49$

$14.3 \pm 8.69$

Herbivores

Herbivores

$0.4 \pm 0.40$

$48.4 \pm$

10.97

Herbivores

$5.2 \pm 3.15$

$21.3 \pm 7.62$

Herbivores

$2.6 \pm 1.66$

$3.8 \pm 1.56$

$3.3 \pm 2.03$

$15.6 \pm$

11.63

$1.4 \pm 1.40$

$0.3 \pm 0.33$

Herbivores

Herbivores

$1.0 \pm 1.00$

$25.4 \pm 19.18$

$7.7 \pm 5.78$

Herbivores

$0.2 \pm 0.20$

$1.2 \pm 0.80$

$1.3 \pm 1.33$

Herbivores

$7.0 \pm 4.63$

Herbivores

$0.4 \pm 0.40$

Herbivores

Herbivores

Herbivores

$6.2 \pm 4.52$

Herbivores

$0.6 \pm 0.60$

Herbivores

$0.8 \pm 0.37$

Herbivores

$1.8 \pm 0.66$

Herbivores

$0.6 \pm 0.24$

Herbivores

$0.2 \pm 0.20$

Herbivores

$0.8 \pm 0.80$

$1.2 \pm 1.20$

$2.8 \pm 1.02$

Herbivores

$0.2 \pm 0.20$

Herbivores

$0.2 \pm 0.20$

Herbivores

Herbivores

$4.8 \pm 2.37$

Herbivores

$1 \pm 0.55$

Herbivores

$0.2 \pm 0.20$
$0.2 \pm 0.20$

$11.2 \pm 4.52$

$9.3 \pm 4.81$

$0.7 \pm 0.67$

$1.2 \pm 1.20$

$2.7 \pm 1.45$

$4.0 \pm 2.02$

$8.3 \pm 4.48$

$0.2 \pm 0.20$

$1.0 \pm 1.00$

$0.6 \pm 0.40$

$4.6 \pm 1.81$

$0.3 \pm 0.33$

$6.7 \pm 3.76$

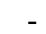

-

$2.7 \pm 1.33$

$0.7 \pm 0.67$

$4.0 \pm 2.08$

$0.3 \pm 0.33$

$0.6 \pm 0.60$

$1.8 \pm 0.66$

$5.0 \pm 4.04$

$2.0 \pm$

$0.3 \pm 0.33$ 


\author{
Siganus corallinus (Valenciennes, 1835) \\ Siganus guttatus (Bloch, 1787) \\ Siganus magnificus (Burgess, 1977) \\ Siganus punctatus (Schneider \& Forster, 1801) \\ Siganus vermiculatus (Valenciennes, 1835) \\ Siganus virgatus (Valenciennes, 1835)
}

\section{Haemulidae}

Diagramma melanacrum (Johnson \& Randall, 2001

Plectorhinchus chaetodonoides (Lacepède, 1801)

Plectorhinchus gibbosus (Lacepède, 1802)

Plectorhinchus vittatus (Linnaeus, 1758)

\section{Lethrinidae}

Lethrinus ornatus (Valenciennes, 1830

Monotaxis grandoculis (Forsskål, 1775)

Monotaxis heterodaon (Bleeker, 1854)

\section{Lutjanidae}

Lutjanus argentimaculatus (Forsskål, 1775)

Lutjanus biguttatus (Valenciennes, 1830)

Lutjanus decussatus (Cuvier, 1828)

Lutjanus fulviflamma (Forsskål, 1775)

Lutjanus fulvus (Forster, 1801)

Lutjanus monostigma (Cuvier, 1828)

Lutjanus quinquelineatus (Bloch, 1790)

Macolor macularis (Fowler, 1931)

Macolor niger (Forsskål, 1775)

\section{Serranidae}

Aethaloperca rogaa (Forsskål, 1775)

Cephalopholis argus (Schneider, 1801

Cephalopholis cyanostigma (Valenciennes, 1828)

Cephalopholis miniata (Forsskål, 1775)

Cephalopholis polyspila (Randall \& Satapoomin, 2000)

Cephalopholis spiloparaea (Valenciennes, 1828)

Cephalopholis urodeta (Forster, 1801)

Epinephelus coeruleopunctatus (Bloch, 1790)

Epinephelus fasciatus (Forsskål, 1775)

Epinephelus merra (Bloch, 1793)

Epinephelus quoyanus (Valenciennes, 1830)

Variola louti (Forsskål, 1775)

\begin{abstract}
Blue-spotted spinefoot
Orange-spotted spinefoot

Magnificent rabbitfish

Goldspotted spinefoot

Vermiculated spinefoot

Barhead spinefoot
\end{abstract}

Blackfin slatey

Harlequin sweetlips

Harry hotlips

Indian Ocean oriental sweetlips

\section{Herbivores \\ Herbivores \\ Herbivores \\ Herbivores \\ Herbivores \\ Herbivores}

$0.2 \pm 0.20$

$0.8 \pm 0.49$

$0.8 \pm 0.80$

$6.8 \pm 5.23$

$-$

$0.8 \pm 0.49$

$0.8 \pm 0.49$

$-$

$1 \pm 0.63$

$0.6 \pm 0.60$

Carnivores

$0.6 \pm 0.60$

Carnivores

Carnivores

Carnivores

$3.2 \pm 3.20$

$2.8 \pm 1.83$

$1.6 \pm 0.93$

$3.3 \pm 3.33$

Ornate emperor
Humpnose big-eye bream
Redfin emperor

Carnivores

$1.2 \pm 0.80$

$2.6 \pm 0.81$

Carnivores

Carnivores

$1.4 \pm 0.75$

$3.8 \pm 1.56$

$2 \pm 2.00$

$1.0 \pm 0.77$

Mangrove red snapper

Carnivores

Two-spot banded snapper

Checkered snapper

Carnivores

Carnivores

Dory snapper

Blacktail snapper

One-spot snapper

Five-lined snapper

Midnight snapper

Black and white snapper

Carnivores

Carnivores

Carnivores

Carnivores

Carnivores

Carnivores

$0.6 \pm 0.60$
$3.4 \pm 1.03$

$0.4 \pm 0.40$

$3.4 \pm 1.03$

$4.4 \pm 1.12$

$1.2 \pm 1.20$

$1.2 \pm 1.20$

$1.8 \pm 1.36$

$0.2 \pm 0.20$

$0.2 \pm 0.20$

Redmouth grouper

Peacock hind

Carnivores

Carnivores

$$
\begin{gathered}
3.4 \pm 0.81 \\
15.2 \pm \\
0.80
\end{gathered}
$$

Carnivores

Bluespotted hind

Coral hind

polyspila hind

Strawberry hind

Darkfin hind

Whitespotted grouper

Blacktip grouper

Honeycomb grouper

Longfin grouper

Yellow-edged lyretail
Carnivores

Carnivores

Carnivores

Carnivores

Carnivores

Carnivores

Carnivores

Carnivores

Carnivores

-

$0.2 \pm 0.20$

$$
\text { - }
$$

$1.2 \pm 0.80$

$0.2 \pm 0.20$

-

$0.2 \pm 0.20$

$0.2 \pm 0.20$

-

$0.4 \pm 0.40$
$0.3 \pm 0.33$

$0.7 \pm 0.67$

$0.3 \pm 0.33$

$10.3 \pm$
$0.3 \pm 0.33$

$2.3 \pm 1.20$

Note: Mean abundance and SE of coral reef fishes for corallivores (Chaetodontidae), herbivores (Acanthuridae, Scaridae, and Siganidae), and carnivore (Haemulidae, Lethrinidae, Lutjanidae, and Serranidae) in Marine Recreational Park: Core Zone (Notake Zone), Utilization Zone (Recreational Zone) and Non-Marine Recreational Park (Fishing Zone) in Pieh per area $350 \mathrm{~m}^{2}$ 


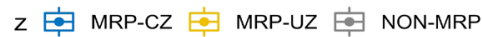

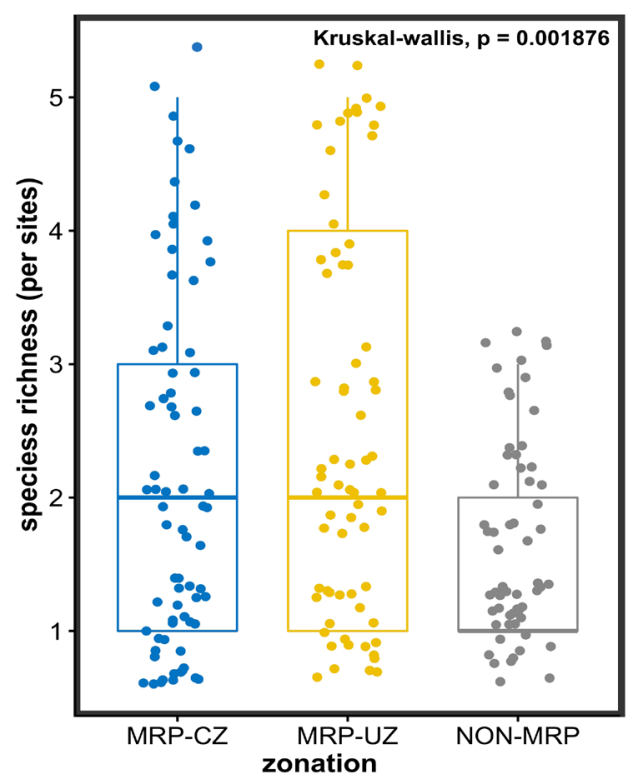

$z$ 间 MRP-CZ 闬 MRP-UZ 闬 NON-MRP

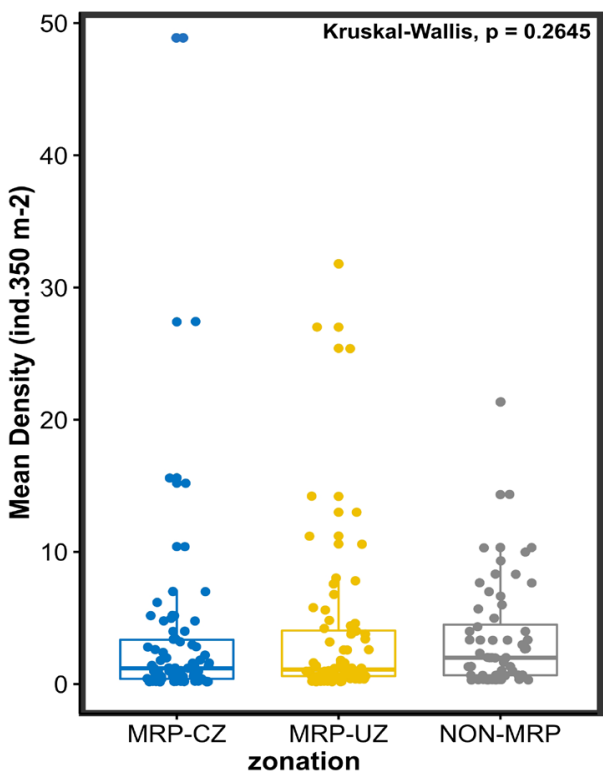

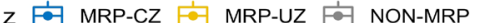

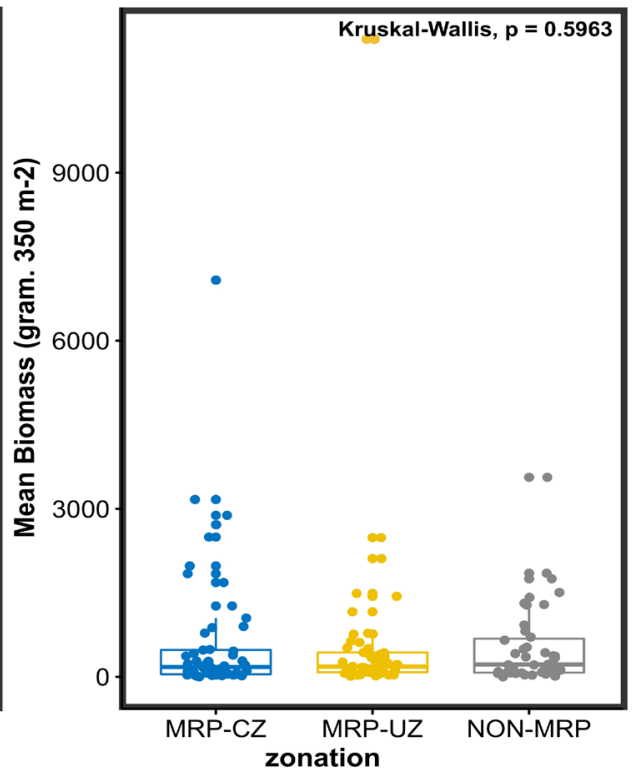

Figure 2. Reef fishes composition (species richness, density, and biomass) in 3 zonation are Pieh MRP (core zone / no-take zone, utility zone, and non - MRP area)
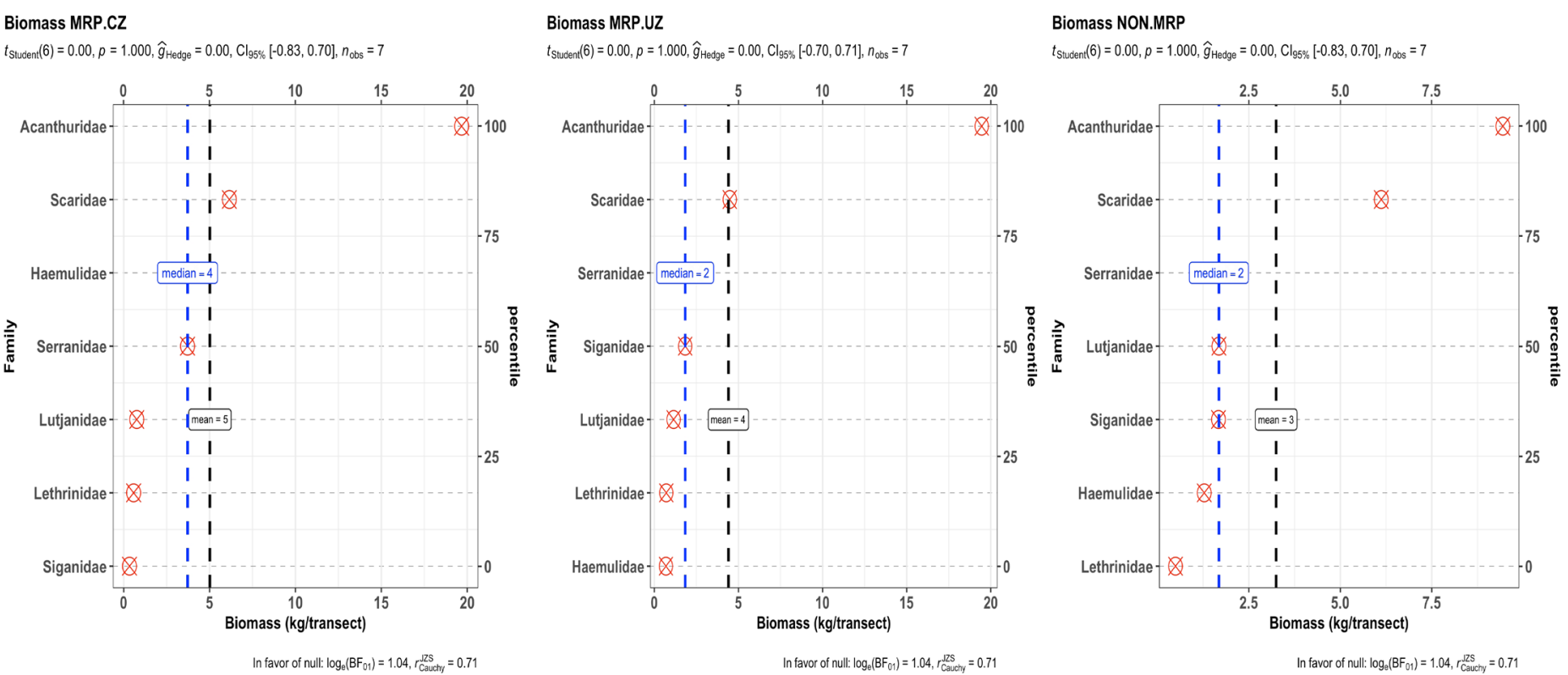

Figure 3. Mean Biomass Rank in 7 Family Coral Reef Fishes (Acanthuridae, Scaridae, Siganidae, Haemulidae, Letrhinidae, Lutjanidae, Serranidae) in Marine Recreational Park for Core Zone (No-take Zone), Utilization Zone (Recreational Zone) and Non-Marine Recreational Zone Park (Fishing Zone) in Pieh (kg/transect)

Based on overall average biomass in different location MRP Area, Acanthuridae represents 55.98\% of the total biomass in MRP-CZ, 63.13\% in MRP-UZ, and $41.55 \%$ in Non-MRP Area (Figure 3). The mean biomass from eight family coral reef fishes in MRP-CZ is higher than MRP-UZ and Non-MRP with $5.02 \mathrm{~kg} /$ transect area (MRP-CZ, $p=0.035, \mathrm{r}=0.83, \mathrm{CI} 95 \%=$ $0.65,1.00, \mathrm{n}=7), 4.40 \mathrm{~kg} /$ transect area (MRP-UZ, $p=$ $0.035, \mathrm{r}=0.83, \mathrm{CI} 95 \%=0.59,1.10, \mathrm{n}=7)$ and $3.25 \mathrm{~kg} /$ transect area (non-MRP, $p=0.022, \mathrm{r}=0.89, \mathrm{CI} 95 \%=$
$0.88, .90, \mathrm{n}=7$ ), respectively (Figure 3 ). Biomass ranking for each MRP location shows that herbivorous fish groups (Acanthuridae, Scaridae) have higher biomasscompared to the carnivorous fish group (Haemulidae, Lethrinidae, Lutjanidae and Serranidae) where at the MRP-CZ location, Acanthuridae has biomass of (19.67 $\pm 6.63) \mathrm{kg} /$ transect area, followed by Scaridae $(6.15$ $\pm 2.48)$, Haemulidae $(3.91 \pm 3.07)$, Serranidae $(3.71 \pm$ $0.44)$, Lutjanidae $(0.76 \pm 0.35)$, Lethrinidae $(0.58 \pm 0.30)$, 
ENS

Location 宁 MRP.Cz 宁 MRP.Uz 宁 NON.MRP
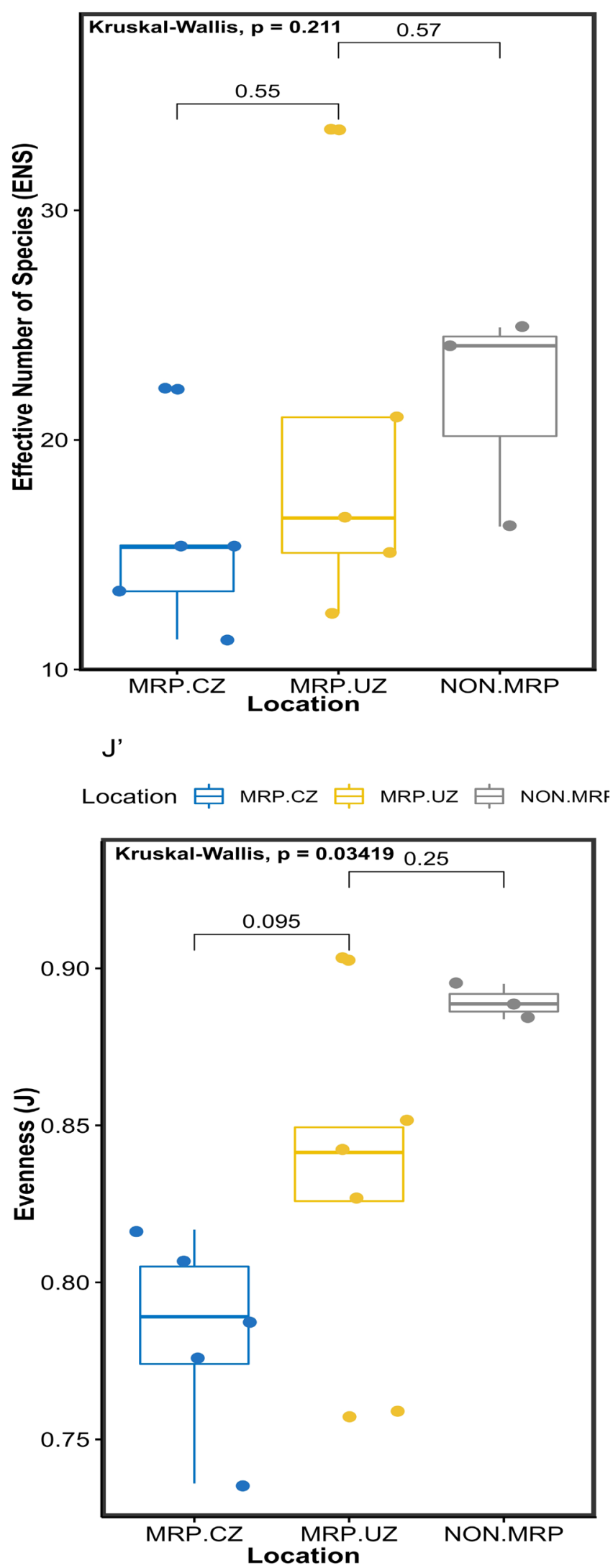

$\mathrm{H}^{\prime}$

Location 户 MRP.CZ 户 MRP.UZ 宁 NON.MRP

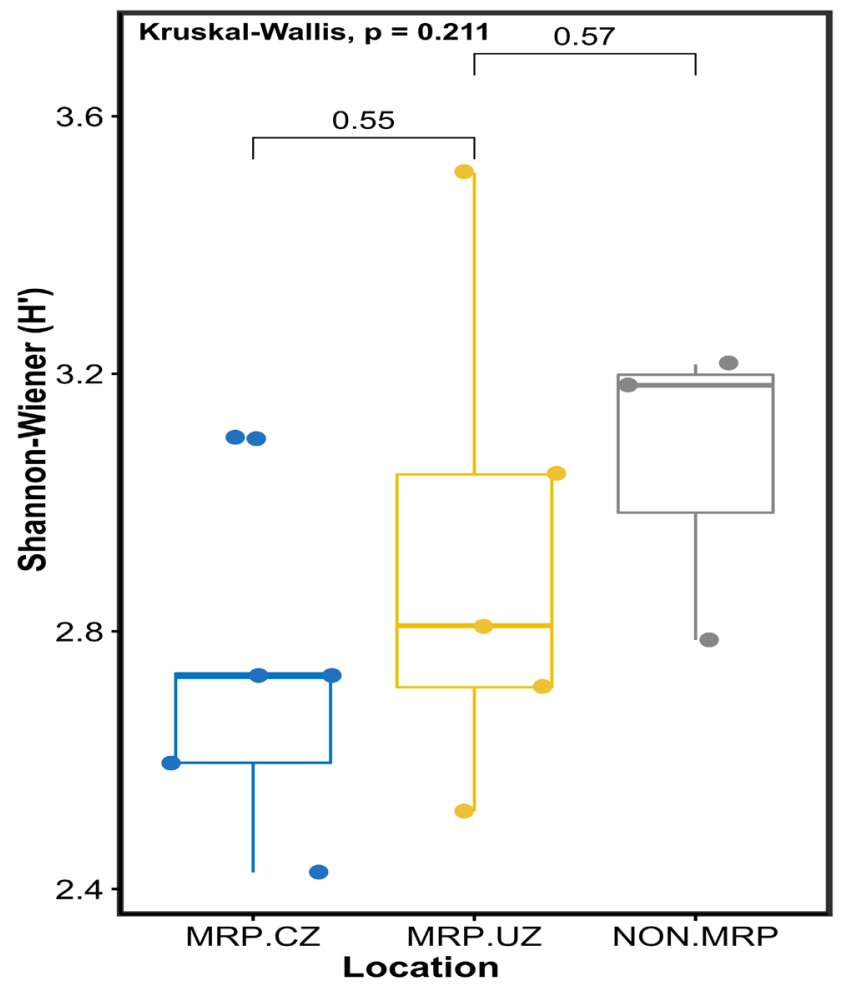

$S$

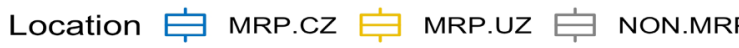

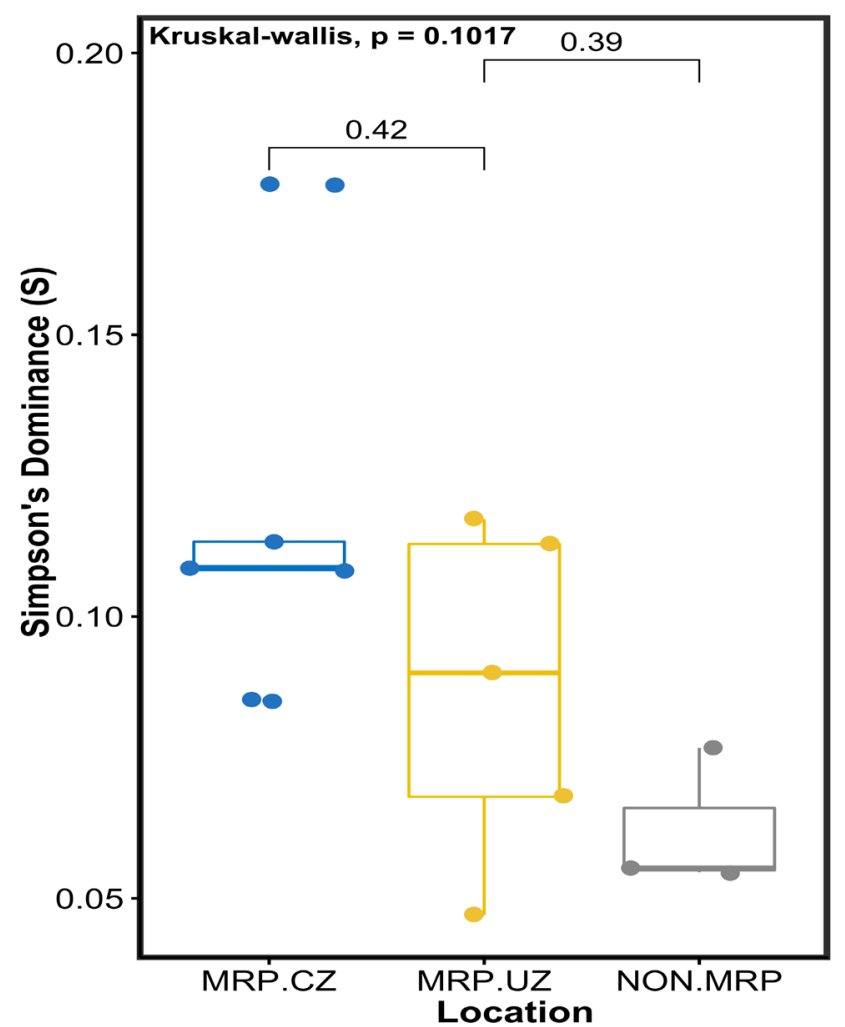

Figure 4. Marine Biodiversity Indices (ENS = Effective Number Species, H' = Shannon-Weaver Index, $S=$ Simpson Dominance, Evenness Equitability (J) between three locations of MRP: Core zone (No-take Zone), Utility Zone (Recreational Zone) and Non-MRP Area (Fishing Zone) in Pieh MRP 


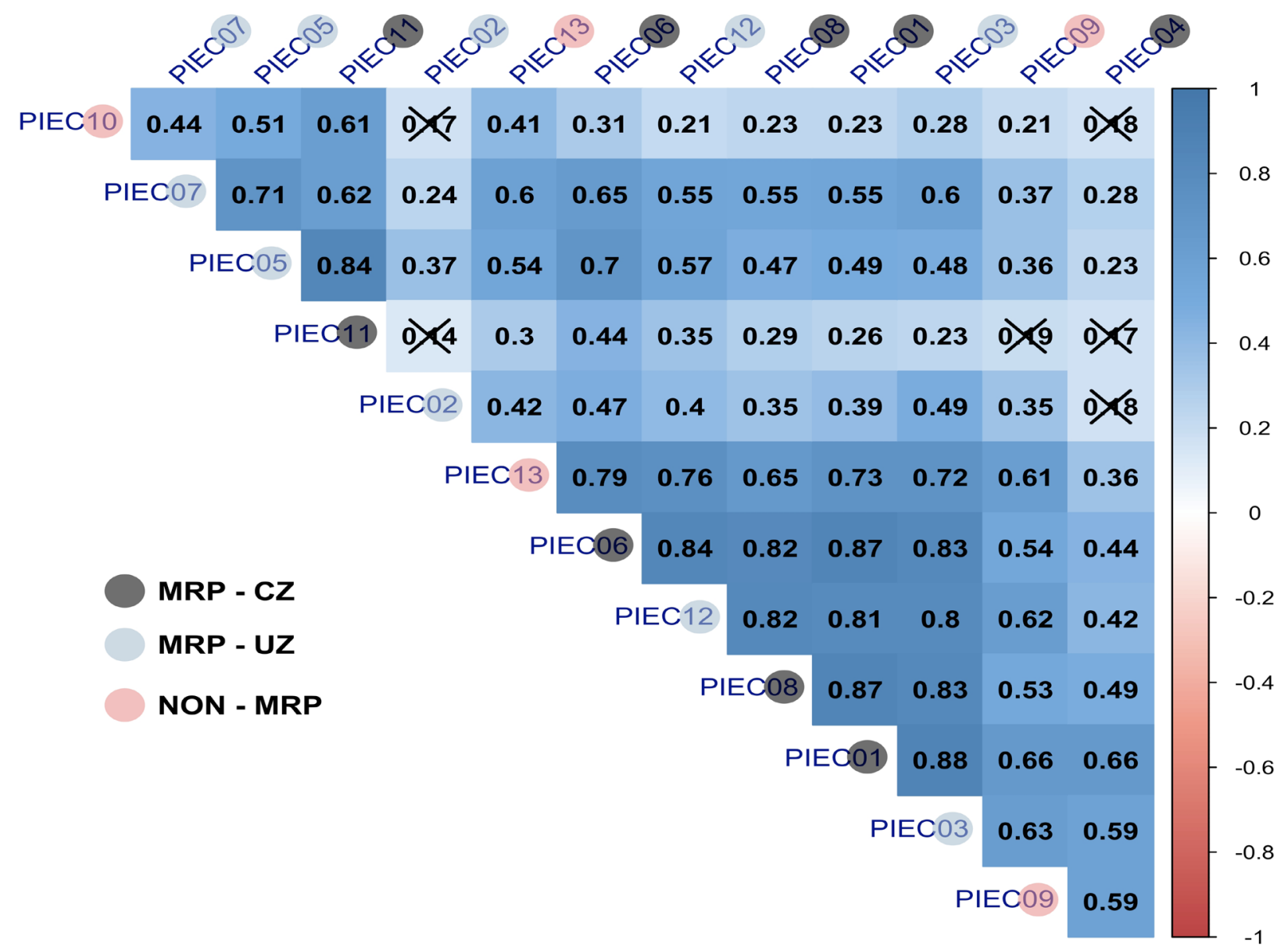

Figure 5. Pearson correlation coefficient matrix with significance level $=0.05$, comparing paired sites per location covariates. Negative correlations are shaded white; positive correlations are shaded grey. The strength of the correlation is indicated by dark grey color saturation. The color of dark grey, red, blue and red in circle shape is also indicated the different locations (MRP-CZ, MRP-UZ, and Non-MRP) where the definition of each covariate (y-axis) and its coded counterpart (upper X-axis) are defined per comparison.
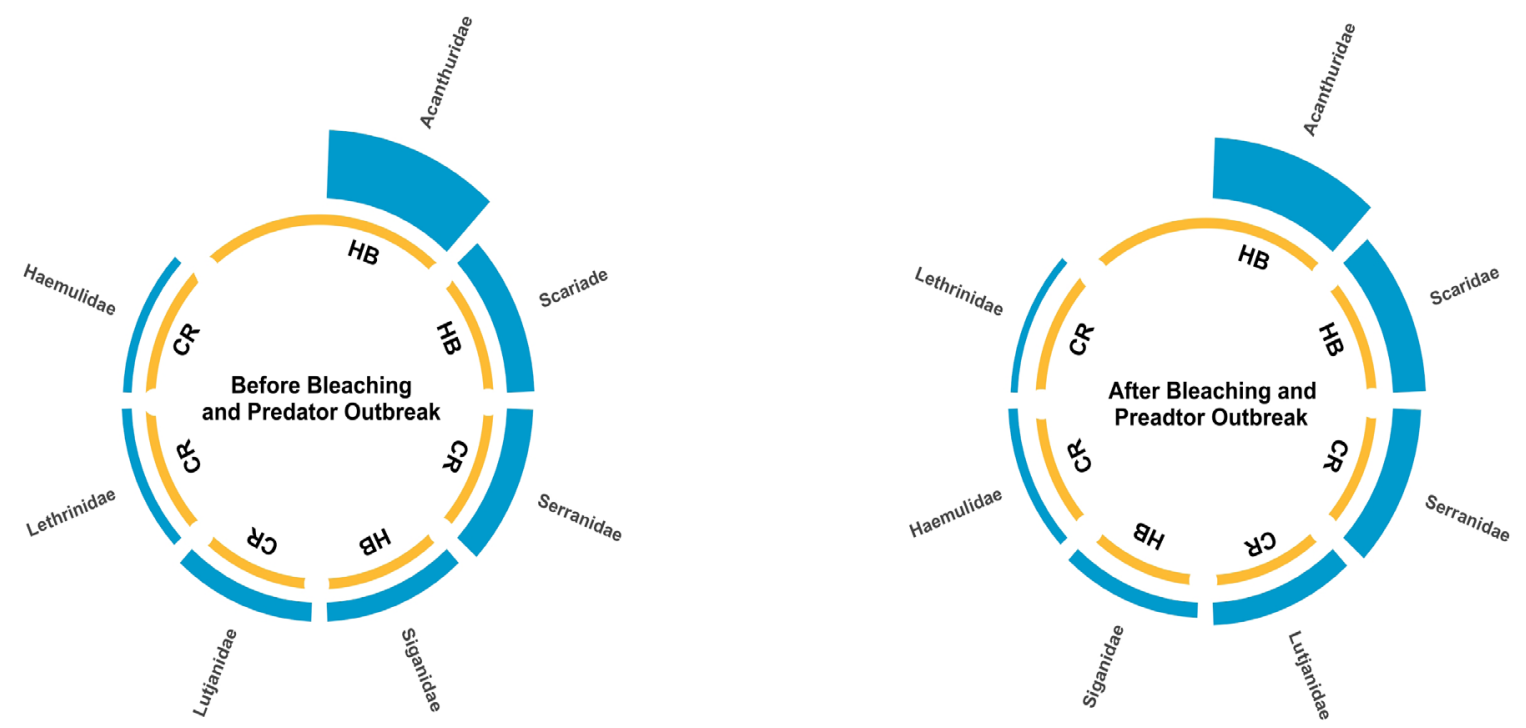

Figure 6. Reef fishes composition before - after bleaching and Acanthaster planci outbreak in Pieh MRP. HB (herbivore), CR (Carnivore) 
and Siganidae $(0.33 \pm 0.27)$. Biomass conditions in the MRP-UZ have an almost similar situation as MRP-CZ, where Acanthuridae is dominant biomass with $19.45 \pm$ $10.32 \mathrm{~kg} /$ transect area, followed by Scaridae $(4.48 \pm$ $1.00)$, Serranidae $(2.59 \pm 0.61)$, Siganidae $(1.83 \pm 1.17)$, Lutjanidae (1.14 \pm 0.43$)$, Lethrinidae $(0.71 \pm 0.20)$, and Haemulidae $(0.69 \pm 0.48)$. Locations in Non-MRP have different conditions with the other two locations with a significantly lower average biomass where Acanthuridae has mean biomass $9.44 \pm 1.33 \mathrm{~kg} /$ transect area, followed by Scaridae (6.12 \pm 2.94$)$, Serranidae $(2.03 \pm$ $1.06)$, Lutjanidae $(1.68 \pm 1.28)$, Siganidae $(1.67 \pm 0.70)$, Haemulidae (1.28 \pm 1.28$)$, and Lethrinidae $(0.50 \pm 0.26)$ (Figure 3).

The indices of diversity from at MRP-CZ / Notake zone area shows an interesting result, the average Shannon index number $(\mathrm{H})$, the Effective Number of Species (ENS), Evenness (J) of coral reef fish on MRP$\mathrm{CZ}$ is lower than location MRP-UZ and Non-MRP. The value of the mean diversity index for the Shannon-Weaver index to MRP-CZ was H' (2.72), ENS (15.53), and J (0.78) (Figure 4). Diversity indices for MRP-UZ are little higher than MRP-CZ, where the value of mean diversity index for Shannon-Weaver index to MRP-UZ was H' (2.92), ENS (19.72) and J (0.84), as for the NonMRP location has the highest diversity indices, where the value of mean diversity index for Shannon-Weaver index to Non-MRP H' (3.06), ENS (21.74) and J (0.89) (Figure 4). Based on measurement by the Simpson's index for each location (MRP-CZ, MRP, UZ, and NonMRP), each location was dominated by a single family of Coral Reef Fishes, apparent from its high value. The dominance of Simpson's index shows, MRP-CZ area has a higher value than MRP-UZ and Non-MRP, where the value of Simpson for MRP-CZ, MRP-UZ, and NonMRP were 0.12, 0.09, and 0.06, respectively (Figure 4). The Kruskal-Wallis test for Shannon- Wiener diversity indices $\left(\mathrm{H}^{\prime}\right)$ (Kruskal-Wallis chi-squared $=3.11$, df $=2$, $\mathrm{p}=0.211$ ) shows no significant difference in diversity in each location. Diversity analysis with Effective Number of Species (ENS) also shows no significant difference between location (Kruskal-Wallis chi-squared $=3.11$, df $=2, p=0.211)$. The equitability analysis with Evenness (J) shows non-MRP location to have significantly higher value than MRP-UZ and MRP-CZ (Kruskal-Wallis chisquared $=6.75, \mathrm{df}=2, \mathrm{p}=0.034)$. The dominance analysis with Simpson's (S) showed no significant difference between each location (Kruskal-Wallis chi-squared = 4.57, $\mathrm{df}=2, \mathrm{p}=0.10$ ) (Figure 4).

Result from Pearson correlation analysis coefficient matrix shows there are some strong positive correlations between each sites location in Marine rec- reational Park Pieh (MRP). Several locations in no-take zone area shows strong positive correlation, including Pandan Island (PIEC.08) and Pieh Islands (PIEC.01) (r $=0.87$ ), Air Island (PIEC.06) and Pieh Island (PIEC.01) $(\mathrm{r}=0.87)$, and Pandan Island (PIEC.08) and Air Island (PIEC.06) $(\mathrm{r}=0.82)$ (Figure 5). Another strong positive correlation shows by no-take zone area and utility zone, including Bando Island (PIEC.01) and Pieh Island (PIEC.03) $(\mathrm{r}=0.88)$, Air Island (PIEC.06) and Toran Island (PIEC.12) $(\mathrm{r}=0.84)$ and Pandan Island (PIEC.08) and Toran Island (PIEC.12) (0.82). In addition, several sites have a weak positive and moderate positive correlation with other sites as non-MRP area (PIEC.10 and PIEC.09) and utility zone, PIEC.07, PIEC.05, PIEC.02, PIEC.09, and no-take zone area (PIEC.04) (Figure 5).

\subsection{Discussion}

The coral bleaching event in 2016 was a global catastrophe on the reefs. Almost in the all tropical or subtropical coral ecosystem reported this phenomenon, including, in Indonesia region (Bachtiar and Hadi, 2019; Ampou et al., 2017; Putra et al., 2019; Wouthuyzen et al., 2018), the Great Barrier Reef (GBR) (Harvey et al., 2018; McMahon et al., 2019; Tebbett et al., 2019; Wismer et al., 2019; Wolanski et al., 2017), Maldives (Nizam et al., 2016), Brazil (Teixeira et al., 2019), Mexico (Johnston et al., 2019), Indian Ocean (Gudka et al., 2018; Head et al., 2019; Ranith and Kripa, 2019; Thinesh et al., 2019), Seychelles (Robinson et al., 2019), Guam (Raymundo et al., 2019), Japan (Nishiguchi et al., 2018), and several other locations that are widespread in the Indo Pacific Ocean. The bleaching phenomena in 2016 in Pieh MRP were largely caused by the increase of Sea Surface Temperature (SST) anomaly due to short duration climate pattern El Niño-Southern Oscillation (ENSO) (Booth and Beretta, 2002). The bleaching event due to El Niño had higher magnitude in 2016 compared to previous events in 2010 (Wouthuyzen et al., 2018) and El Niño's effects are worse due to the long-term global warming (Baird et al., 2009; Hughes et al., 2003) and climate changes (Baker et al., 2008; Hughes et al., 2018). After the bleaching event, the Pieh MRP location hit by $A$. planci outbreak, and there is no evidence why an outbreak of coral predators occurred in the Pieh MRP location after the bleaching event. There was a strong suggestion that the outbreaks of $A$. planci in Pieh MRP were due to warmer sea surface temperatures and higher nutrients (Wouthuyzen et. al., 2020). Previous study documented from Haywood et al. (2019), the pattern of $A$. planci outbreaks have occurred after bleaching in Pilbara offshore bioregion. However, the reason on why this pattern can occur in Pieh MRP has not yet discovered. It can only be ensured that suitable habitat conditions and food resources on the reef can support an outbreak of $A$. planci. The high population of $A$. planci 

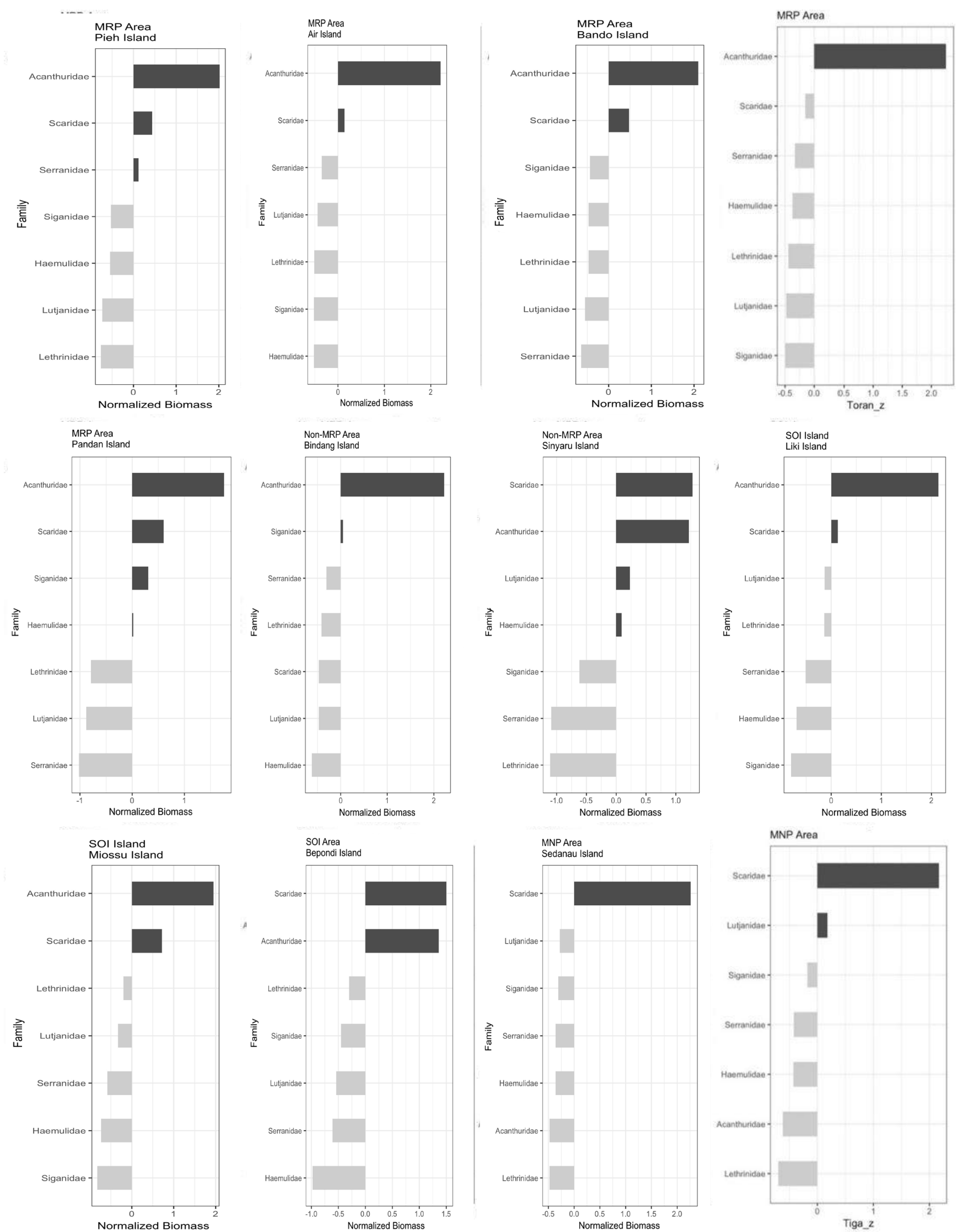

Figure 7. Diverging Bars of Biomass Coral Reef Fishes from Herbivore group (Acanthuridae, Scaridae, Siganidae) and Carnivore group (Haemulidae, Lethrinidae, Lutjanidae, Serranidae) in some oceanic Island in Indonesia; Pieh Area (Pieh island, Air island, Bando island, Toran island, Pandan island, Bindalang island, Sinyaru island); Papua area (Liki island, Miossu island, Bepondi island); Natuna area (Sedanau island, Tiga island). MRP (Marine Recreational Park); SOI (Smalls Outer Island); MNP (Marine National Park). Dark Grey is above the average of Biomass; Light Grey is below the average of biomass. 
is the response to seasonal variation in temperature and storm-generated by wave (Haywood et al., 2019), and increase in the concentration of chlorophyll- $a$ as food availability for $A$. planci larvae due to upwelling process that transport rich-nutrient to coral ecosystem (Baker et al., 2008), but Pratchett et al. (2017) explained the $A$. planci outbreak phenomenon still cannot be explained and remains mostly unresolved. In current study, it is aimed to highlight no significant change in the fish composition after the bleaching and A. plan$c i$ outbreak in Pieh MRP (Figure 6). Before and after non-anthropogenic disturbance events, the composition of fish in Pieh MRP is still dominated by herbivores fishes (Acanthuridae and Scaridae), followed by Serranidae as carnivore fish. (Figure 6).

The similar fish composition before and after disturbance in Pieh MRP shows the coral reeffish ill endurance on bleaching event and $A$. planci outbreak, but the coral reef ecosystem requires full recovery condition. Current study result suggested the need for integrated management after post-disaster in Pieh MRP due to the possibility of accelerated recovery in the coral reef ecosystem after post-disaster for the next future event. The possibility of accelerated recovery condition after coral bleaching and Acanthaster outbreak in Pieh MRP enhanced by geographical condition and physical environment that reduce the temperature of the sea (Morgan et al., 2017; West and Salm, 2003). Pieh MRP is a group of islands facing the Indian Ocean and included in the category of open ocean exposure (OOE) islands. The OOE islands usually present a coral reef system that is strongly influenced by strong waves and currents (Teixeira et al., 2019), which in turn supports the occurrence and abundance of planktivorous (Floeter et al., 2004; Pinheiro et al., 2011) and reduce bleach event (Baker et al., 2008). Most of the small Island condition Pieh MRP had a deeper reef with a reef slope near $90^{\circ}$ (Figure 1) (Abrar et al., 2014). The topography of reef slope potential had the strength of upwelling conditions and some locations in western Indonesia at the Indian Ocean region has generally had the strength of upwelling condition as Mentawai Island(Abram et al., 2003). The reef slope condition in open ocean exposure islands can change dramatically to reduce the high temperature due to the impact of cold-water upwelling conditions and the previous study by Wouthuyzen et al. (2018) explained that strong cold-water upwelling could assist in the recovery process from coral bleaching event in the Indian Ocean. Another oceanographic phenomenon that allows Pieh MRP accelerated recovery in bleaching coral was strong current and waves, where the rapid mass of water flow can protect coral from bleaching event by removing harmful oxygen radical (Grimsditch and Salm, 2006). The accelerated recovery of coral reefs in Pieh MRP was also influenced by the dominant composition of herbivorous fish. Herbivore fish has an essential role after the disturbance by reducing algal (McManus and Polsenberg, 2004). An interesting result of this study, all small outer islands that are facing the ocean (OOE) have a similar fish composition pattern as herbivorous fish dominate in coral reef ecosystems. Previous studies show the outer island and OOE can provide a large presence roving herbivores fish (Friedlander and DeMartini, 2002; Pinheiro et al., 2015; Sandin et al., 2008b). The large presence of herbivorous fish also plays a role in maintaining the sudden growth of macroalgae after bleaching. Based on current study, it has found several important herbivore fish species as macroalgal remover in Pieh MRP which are Naso unicornis and Chlorurus sordidus, both were recorded feeding on macroalgal in Seychelles Islands (ChongSeng et al., 2014); and Zebrasoma veliferum has been recorded feeding on macroalgal in Great Barrier Reef (Hoey and Bellwood, 2011). The presence of these species is expected to help the recovery process in Pieh, especially from macroalgae.

After bleaching disturbance and A.planci outbreaks, herbivore fishes species were dominated (60.73 $\%$ ) in Pieh MRP, including the three most abundant species were Ctenochaetus striatus, A. lineatus and $N$. hexacanthus (Table 2 and Figure 3). The dominance of these herbivores fishes group species contributes to the higher biomass in all area in Pieh MRP: core zone (no-take zone), utility zone (recreational zone), and nonMRP (fishing zone) (Figure 3). The presence of herbivorous fish after a non-anthropogenic disturbance provides important information on the resilience of coral reef ecosystems in Pieh MRP. Herbivorous fish have an important role in coral reef resilience after climate change phenomena (Hughes et al., 2007) including coral bleaching and $A$. planci outbreaks due to their ability to control blooms of turf algal and fleshy seaweeds and support coral recruitment and growth (Mumby et al., 2006; 2007). The mass-bleaching coral events may result in massive algal overgrowth (Diaz-Pulido and McCook 2002) and algae become dominant in competitive interaction when the coral colonies suffer from bleaching (Swierts and Vermeij, 2016). The condition after disturbance is an essential indicator of understanding the resilience of the coral ecosystem and the presence of herbivorous fish can strengthen the resilience. The result of current study showed that after catastrophic disturbance herbivore fish composition (species richness, abundance, and biomass) was higher within the no-take area (core zone) than in utility zone and non-MRP area (Table 2). This finding was consistent with previous studies that suggested biomass of herbivorous fishes is higher within MPAs area than in non-protected areas (Graham et al., 2007; Wilson et al., 2012) and the fishing practice was a major cause to present the low composition of herbivorous fish in the non-MPA region. If several herbivorous fishes species 
from Scaridae, Siganidae, and Acanthuridae family continues to be fishing objects after catastrophic disturbance (coral bleaching and $A$. planci outbreak), it will have a significant impact on the recovery process on coral reefs in Non-MRP Pieh area.

The dominant presence of herbivorous fish, $C$. striatus, A. lineatus, and $N$. hexacanthus after non-anthropogenic disturbance in the Pieh MRP region provide valuable information for the recovery process of coral reef ecosystems condition after post-disaster (Table 2 and Figure 3). This family group of fish has an essential part of its diet consisting of detritus and calcified ingredients (Scaridae and Acanthuridae); the abundance and distribution of these fish are strongly influenced by warmer locations (Floeter et al., 2004). This confirms the process of increasing water temperatures in 2016 and 2017 in Pieh MRP that resulted in coral bleaching which in turn increased the abundance of this group of fish. The dominance of this group is also strengthened by the coverage of benthic of the coral reef area, which is dominated by Dead Coral Algae and rubble which are primary components diet for these fish groups to grow. The increase in the number of herbivorous fish groups in Pieh MRP indicated a dramatic increase in the representation of dead corals and rubble. The case of mass coral bleaching in 2016 at Pieh MRP resulted in a significant decrease in live coral cover and an increase in dead corals. The research from Putra et al. (2018) explained that there was a positive correlation of biomass of herbivorous fish with dead coral with algae (DCA), especially for herbivorous fish in the Scaridae family, and Sandin et al. (2008a) found there was a significant positive correlation between herbivorous biomass and macroalgae cover, but Newman et al. (2006) and Sandin, et al. (2008b) noticed there was a negative correlation between herbivorous fish and fleshy algae. The Pieh MRP is dominated by Acanthuridae especially from C. striatus. On the coral reef ecosystem, C. striatus plays a critical role in several key ecosystem processes (Tebbett et al., 2018) and has a contribution to remove the epilithic algal matrix (EAM) or algal turf (Tebbett et al., 2017). Additionally, this study found several other important herbivorous fish feeding EAM beside C. striatus, including Scarus ghobban, $S$. dimidiatus, S. niger, S. oviceps, S. rubroviolaceus, Chlorurus bleekeri, and all these fishes have been identified consumer EAM (Bellwood et al., 1990; Vergés et al., 2012). Based on the previous study, the colonization of EAM or algal turf in coral was a consequence of bleaching events and responsible for coral mortality (Diaz-Pulido and McCook, 2002). The interaction of the turf algal and coral causes tissue damage and decrease in pigmentation in coral (Wild et al., 2014) due to turf algae acting as poison to scleractinian coral and able to kill coral tissue (Jompa and McCook, 2003; Titlyanov et al., 2007). Brown et al. (2018) suggested that low turf algal coverage indicates a healthy coral reef. The presence of $C$. striatus can prevent massive coral deaths due to tissue damage by algae turf after the catastrophic coral bleaching. This fish consumes more intensively on sparse/short algae turf and significantly removes more algae turf per hour than other herbivore fish from the Acanthuridae family (Marshell and Mumby, 2012).

In current study, it has found several groups of herbivorous fish from A.tristis, C. striatus, C. binotatus, and $S$. niger species with less than $5 \mathrm{~cm}$ in size which indicates an increase in number and high regeneration process of herbivore fish groups in Pieh MRP. The increased herbivorous grazing in MPAs results in substantial reductions in algae and encourage coral recovery (Mellin et al., 2016; Mumby et al., 2006), but in reality, the herbivorous fish populations are strongly influenced by region. Current study's results show that after bleaching and $A$. planci outbreak, the mean abundance of C. striatus was two times higher in the core zone / no-take area $(48.4 \pm 10.97)$ than non-MRP / fishing zone area (21.3 \pm 7.62$)$ (Table 2). Although C. striatus is one of the most abundant surgeonfish in the Indo-Pacific coral reef, but their existence and population could be threatened because large environmental disturbances (Lin et al. 2021) or several fishing practice (Jones et al., 2004). However, this is a fundamental problem with the resilience process. If $C$. striatus and several herbivorous fish populations become lower in number and provide low grazing pressure to algae, herbivores may not be able to resist increasing algal population and macroalgae bloom (Hughes et al., 2007; Pratchett et al., 2011). Unfortunately, it brings a rapid phase in changing from coral-dominated to algal-dominated (Pratchett et al., 2011). The increasing number of dead coral algae (DCA) serves as herbivore fish food source and causes a dramatic increase in the grazing group of herbivore fish (Jones et al., 2004; Putra et al., 2018). In addition, herbivore fish populations can still play an essential role in performing the function of regeneration in the shifting phase of the reef, which is dominated by algae. Grazing herbivorous fish helps maintain the health of coral reefs dominated by algae (Bellwood et al., 2004; Thibaut and Connolly, 2013). The only significant correlation noted between the benthic and fish assemblages was a positive relationship between herbivorous fish biomass and macro-algal cover (Putra et al., 2018; Sandin, et al., 2008b). It is most detrimental if phase shift from coral-dominated to algal- dominated are not able to regenerate optimally and apprehended the reefs without coral, it will no longer support diverse reef fish but will be dominated numerically by a small group of reef fish species that prefer algae or rubble (Jones et al., 2004). A basic understanding of the phase shift from coral-dominated to the algal-dominated mechanism that informs coral reef degradation will affect coral reef fish communities differently but limited time scales. The 
long-term effects of reducing coral cover in coral reef fish communities can arise through mechanisms that don't have a direct impact on demographic levels such as mortality but change regularly and cause a significant reduction in the physiological conditions of the population (Feary et al., 2009).

Other findings from current study showed, after the disturbance, the number of species and populations from corallivore fishes (Chaetodontidae) has decreased. Still, there has been an increase in carnivorous fish populations (Table 3). Several species of corallivore fishes (Chaetodontidae) were not found at the time of the study, including Chaetodon bennetti (obligate corralivore), C. xanthocephalus (Facultative corralivore), and Heniochus diphreutes (generalist corralivore). This study's result showed, more than two years, the population of corallivores fishes has reduced from $(38 \pm$ $3.59)$ in 2014 to $(31 \pm 4.13)$ in 2019 (Table 3). The reduction of corallivores fish composition caused by nutritional deficit and decrease in aggressive behavior among corallivore fish due to after bleaching event (Keith et al., 2018). The aggression behavior provides information changing the population of corallivores fishes due to competing individuals in obtaining enough food resources (Tricas, 1989; Yahya et al., 2011) and influence in reducing butterflyfish abundance (Pratchett et al., 2006) in the long term (> 4 weeks) lethal or sub-lethal effect of feeding form (Cole et al., 2009). In short term, corallivores are found to impose further stress and increase the feeding rate on bleach coral (Cole et al., 2009) and may contribute to increase mortality of bleached corals. If it lasts longer, food sources are limited and may contribute in reducing in the abundance of corallivore fish. On the other hand, the population of carnivore fish increased more than 20 percent and biomass increased more than 30 percent (Table 3). Explosion of herbivorous fish populations after bleaching and A. planci outbreaks provide a substantial food source for carnivorous fish. Current study shows, after the bleaching and A. planci outbreak, the population of herbivorous fishes had increased $(6.77 \pm 0.78)$.
The increased population of herbivorous provides food source for carnivorous fishes which increased carnivorous fish population and biomass (Table 3). C. striatus contributed as the highest food source for several carnivorous fish, especially for $C$. argus. In current study, C. argus is the dominant species for the carnivorous group. After the disturbance, the population of C. argus increased the abundance of $C$. striatus (Table 2). A previous study showed $16.9 \%$ (Hawaii) composition of the fish portion of the diet of C. argus was Acanthuridae family with \%IRI = 20.9 (Dierking et al., 2009). The diet of $C$. argus is dependent on considering the prey availability and influence on prey composition (Meyer and Dierking, 2011). The high dominance of C. striatus (Quoy \& Gaimard, 1825) (Acanthuridae) and another herbivorous group after disturbance in Pieh MRP impact on an increase in population C. argus.

Biomass and the diversity of reef fish have an essential role in maintaining the structure and resilient process of coral reefs (Chong-Seng et al., 2014; McClanahan et al., 2011; Thibaut and Connolly, 2013). The results of the study showed MRP area has higher biomass than the non-MRP area (Figure 3). The non-MRP area is a location that is permitted for fishing activities by local fishermen. Consequently, this area has an impact on reducing fish biomass, especially economically targeted fish from carnivorous groups (Haemulidae, Lethrinidae, Lutjanidae, Serranidae) (Table 2). Location with the highest fish biomass is mostly within the boundaries of marine protected areas, suggesting that human exploitation is a major factor in reducing fish biomass (Sandin et al., 2008a). The core zone / no-take zone and utilization zone in the MRP area have almost similar mean biomass value of reef fish. This explains the marine recreational activities in utilization zone Pieh MRP have no impact on reducing fish biomass and this also confirms that the management of the MRP area in Pieh by the government has been correctly implemented. The overall findings indicate that the highest reef fish abundance and biomass were in the MRP zone management control area. The results of this study also show that

Table 3. The changing coral reef composition before (2014) and after (2019) bleaching phenomena and Acanchaster planci outbreaks

\begin{tabular}{ccccccc}
\hline Condition & $\begin{array}{c}\text { Corralivore } \\
\text { Species Rich- } \\
\text { ness (SR) }\end{array}$ & $\begin{array}{c}\text { Mean abun- } \\
\text { dance } \pm \mathbf{s e}\end{array}$ & Mean SR $\pm \mathbf{s e}$ & $\begin{array}{c}\text { Mean bio- } \\
\text { mass } \pm \mathbf{s e}\end{array}$ & $\begin{array}{c}\text { Mean SR } \\
\pm \mathbf{s e}\end{array}$ & $\begin{array}{c}\text { Mean Bio- } \\
\text { mass } \pm \mathbf{s e}\end{array}$ \\
\hline Before Pressure (2014) & 21 & $38 \pm 3.59$ & $4.10 \pm 0.70$ & $6.66 \pm 1.47$ & $5.70 \pm 0.87$ & $4.34 \pm 0.88$ \\
\hline After Pressure (2019) & 17 & $31 \pm 4.13$ & $6.77 \pm 0.78$ & $6.40 \pm 1.23$ & $7.69 \pm 0.85$ & $7.01 \pm 1.50$ \\
\hline
\end{tabular}

Note: The Corallivore counted for Chaetodontidae family; Herbivore (Scaridae and Siganidae)

family; Carnivore Fish (Haemulidae, Lethrinidae, Lutjanidae, Serranidae) family. Mean abundance

(individual $/ 350 \mathrm{~m} 2)$ and mean biomass $(\mathrm{kg} / 350 \mathrm{~m} 2$ ). 
management of control in the MRP area must be part of a future coral reef management strategy. Not only in Pieh MRP but in several other locations that have a high potential of marine tourism can hold integrated controls between conservation and recreation to create sustainable marine tourism (Madduppa et al., 2014; Rudi et al., 2014). The process of restoring coral reef ecosystems caused by post-bleaching phenomena and massive predators of $A$. planci on the Pieh MRP area could be executed by implementing excellent management, including reducing recreational activities in utility zone in the MRP area (Hughes et al., 2003). When the appearance of benthic fleshy algae has decreased dramatically, the presence of large predators from car-nivorous fish family (Haemulidae, Lethrinidae, Lut-janidae, Serranidae), and recovery in the population of corallivorous fishes in the Pieh MRP area, this indicator shows that in Pieh MRP area has contributed recovery from post-bleaching and massive predator of A. planci outbreaks and provides a good indicator for coral reef health (Chabanet et al., 2016).

The coral reef recovery process after non-anthropogenic disturbance must be assisted with integrated management of Pieh MRP, especially those related to tourism activities in the MRP area. Tourism contributes to several benefits. Proper tourism management contributes to increasing the economy of the community. Some of the benefits of tourism development include increasing employment opportunities, increasing economic income, increasing population living standards, and promoting culture (Wu, 2014). On the other hand, if tourism development not appropriately managed, it can make a significantly negative contribution, especially to the environment. Comparative research on recreational zones involved in diving and non-diving activities on coral reefs proves that diving with SCUBA has a significant impact on the area of coral reefs visited, especially on hard corals (Tratalos and Austin, 2001) where $15 \%$ of diving activities give damage on coral reefs, with diving fins being the leading cause (95\%) of all damage (Rouphael and Inglis, 1997). In addition to implementing restrictions on activities in the utilization zone, providing qualified control in recreational activities is particularly critical at Pieh MRP. SCUBA diving activities at high recreational levels affect coral communities through direct contact by divers from SCUBA equipment on sensitive benthic organisms (Hammerton, 2017; Luna et al., 2009). Snorkeling and SCUBA activities in the MRP area significantly change benthic topographic features and cause physical damage to coral polyp organisms which are the primary organisms as health indicator of coral reef ecosystems and over the past two decades, there is increasing evidence that many of the biological and aesthetic were damaged by recreational diving (Hammerton, 2017).

\section{Conclusion}

The Biomass and the diversity of reef fish have an essential role in maintaining the structure and process of coral reefs. The herbivorous fish is the most dominant group in the locations of Pieh MRP and NonMRP. After disturbance in Pieh MRP, the coral reef fishes still shows endurance after the bleaching and $A$. planci outbreaks. However, the composition of corallivorous has decreased while herbivorous and fish populations (diversity, abundance and biomass) has increased. The herbivore fishes could shift algal reef assemblages to states that are beneficial for corals and improve corals' ability to thrive, thereby increasing corals' ability to recover from destructive events such as bleaching and $A$. Planci outbreaks. It is a necessity to restore the condition of coral reefs in the MRP area of Pieh from the phenomenon of mass coral bleaching and attack by A. planci. Management strategy is important to protect herbivorous fish and other fish by constricting supervision at Pieh MRP. In addition, proactive management of the Pieh MRP aims to ensure sufficient stock of herbivores before the re-occurrence of future bleaching events. With sufficient stocks of herbivory in Pieh, the condition of coral reefs in Pieh MRP will recover, although it will take several years. This study suggests that herbivore management is part of a broader strategy to manage and reduce threats to coral reefs in Pieh.

\section{Acknowledgement}

This study was funded by the Coral Reef Rehabilitation Management Program (COREMAP) and supported by data Nusa Manggala. The authors thank Minang Bahari NGO and Directorate General of Treasury Affairs, Minister of Maritime Affairs and Fisheries, Pekanbaru for a helpful collaboration and teamwork for this study. I also thank Prof. Suharosono for giving us some suggestions and information.

\section{Authors' Contributions}

All authors have contributed to the final manuscript. The contributions of each author are as follows, Risandi, Rikoh, and Abrar; collected the data, compiled the manuscripts, and designed the visualization from $\mathrm{R}$ software. Risandi; made a graphic visualization of the data analysis. Ni Wayan Puranmasari, and Jayedul; helped to compile information related to the research. All authors discussed the results and contributed to the final manuscript.

\section{Conflict of Interest}

The authors declare that they have no competing interests. 


\section{Funding Information}

This research was partially supported by COREMAP-CTI Monitoring Program in MRP Pieh 2019

\section{References}

Abelson, A., Nelson, P., Edgar, G., Shashar, N., Reed, D., Belmaker, J., Krause, G., Beck, M., Brokovich, E., France, R., \& Gaines, S. (2016). Expanding marine protected areas to include degraded coral reefs. Conservation Biology, 30(6):1182-1191.

Abram, N. J., Gagan, M. K., McCulloch, M. T., Chappell, J., \& Hantoro, W. S. (2003). Coral reef death during the 1997 Indian Ocean dipole linked to Indonesian wildfires. Science, 301(5635):952-955.

Abrar, M., Giyanto, Siringoringo, R., Edrus, I., Arbi, U., Sihaloho, H., Salatalohy, A., \& Sutiadi. (2014). Laporan monitoring (baseline) kesehatan ekosistem terumbu karang dan ekosistem terkait lainnya - Taman Wisata Perairan Pulau Pieh dan sekitarnya, Sumatera Barat (1 ${ }^{\text {st }}$ ed.). Jakarta: CRITC COREMAP-CTI LIPI.

Allen, G., Steene, R., \& Humann, P. N. D. (2003). Reef fish identification - tropical Pacific fishes Condensed. New World Publicatons, Inc.

Ampou, E. E., Johan, O., Menkes, C. E., Niño, F., Birol, F., Ouillon, S., \& Andrefouet, S. (2017). Coral mortality induced by the 2015-2016 El-Niño in Indonesia: The effect of rapid sea level fall. Biogeosciences, 14(4):817-826.

Attamimi, N. R. (2019). Coral reefs degradation pattern and its exposure towards climate change in Bunaken National Park. International Journal of Geomate, 17(60):170-175.

Bachtiar, I., \& Hadi, T. A. (2019). Differential impacts of 2016 coral bleaching on coral reef benthic communities at Sekotong Bay, Lombok Barat, Indonesia. Biodiversitas, 20(2):570-575.

Baird, A. H., Bhagooli, R., Ralph, P. J., \& Takahashi, S. (2009). Coral bleaching: the role of the host. Trends in Ecology and Evolution, 24(1):1620.

Baker, A. C., Glynn, P. W., \& Riegl, B. (2008). Climate change and coral reef bleaching: An ecological assessment of long-term impacts, recovery trends and future outlook. Estuarine, Coastal and Shelf Science, 80(4):435-471.

Bellwood, D. R., Hughes, T. P., Folke, C., \& Nyström,
M. (2004). Confronting the coral reef crisis. Nature, 429(6994):827-833.

Bellwood, David R., \& Choat, J. H. (1990). A functional analysis of grazing in parrotfishes (family Scaridae): The ecological implications. 189214.

Booth, D. J., \& Beretta, G. A. (2002). Changes in a fish assemblage after a coral bleaching event. $\mathrm{Ma}$ rine Ecology Progress Series, 245:205-212.

Bouchon-Navaro, Y. (1981). Quantitative distribution of the Chaetodontidae on a reef of Moorea Island (French polynesia). Journal of Experimental Marine Biology and Ecology, 55(2-3):145157.

Brown, K. T., Bender-Champ, D., Kubicek, A., van der Zande, R., Achlatis, M., Hoegh-Guldberg, O., \& Dove, S. G. (2018). The dynamics of coral-algal interactions in space and time on the southern Great Barrier Reef. Frontiers in $\mathrm{Ma}$ rine Science, 5:1-13.

Bruno, J. F., \& Valdivia, A. (2016). Coral reef degradation is not correlated with local human population density. Scientific Reports, 6:1-8.

Buxton, C. D., \& Smale, M. J. (1989). Abundance and distribution patterns of three temperate marine reef fish (Teleostei: Sparidae) in exploited and unexploited areas off the Southern Cape Coast. The Journal of Applied Ecology, 26(2):441-451.

Chabanet, P., Bigot, L., Nicet, J. B., Durville, P., Massé, L., Mulochau, T., Russo, C., Tessier, E., \& Obura, D. (2016). Coral reef monitoring in the Iles Eparses, Mozambique Channel (20112013). Acta Oecologica, 72:62-71.

Cheal A. J., Wilson. S. H., Emslie, M. J., Dolman, A. M., \& Sweatman, H. (2008). Responses of reef fish communities to coral decline on the Great Barrier Reef. Marine Ecology Progress Series 372:211-223.

Chong-Seng, K. M., Nash, K. L., Bellwood, D. R., \& Graham, N. A. J. (2014). Macroalgal herbivory on recovering versus degrading coral reefs. Coral Reefs, 33(2):409-419.

Cole, A. J., Pratchett, M. S., \& Jones, G. P. (2009). Effects of coral bleaching on the feeding response of two species of coral-feeding fish. Journal of Experimental Marine Biology and Ecology, 373(1):11-15.

Corrales, C. M., Delan, G. G., Rica, R. L. V., Piquero, A. S., \& Monte, I. A. (2015). A baseline study on coral reef fishes in the marine protected areas 
in Southern Cebu, Philippines. Tropical Technology Journal, 19(1):1-8.

De Freitas, D. M., Sutton, S. G., Moloney, J. M., Lédée, E. J. I., \& Tobin, R. C. (2013). Spatial substitution strategies of recreational fishers in response to zoning changes in the Great Barrier Reef Marine Park. Marine Policy, 40(1):145153.

Diaz-Pulido, G., \& McCook, L. (2002). The fate of bleached corals: patterns and dynamics of algal recruitment. Marine Ecology Progress Series, 232:115-128.

Dierking, J., Williams, I. D., \& Walsh, W. J. (2009). Diet composition and prey selection of the introduced grouper species peacock hind (Cephalopholis argus) in Hawaii. Fishery Bulletin, 107(4):464-476.

Feary, D. A., McCormick, M. I., \& Jones, G. P. (2009). Growth of reef fishes in response to live coral cover. Journal of Experimental Marine Biology and Ecology, 373(1):45-49.

Floeter, S. R., Ferreira, C. E. L., Dominici-Arosemena, A., \& Zalmon, I. R. (2004). Latitudinal gradients in Atlantic reef fish communities: Trophic structure and spatial use patterns. Journal of Fish Biology, 64(6):1680-1699.

Friedlander, A. M., \& DeMartini, E. E. (2002). Contrasts in density, size, and biomass of reef fishes between the northwestern and the main Hawaiian Islands: The effects of fishing down apex predators. Marine Ecology Progress Series, 230:253-264.

Froese, R., \& Pauly, D. (2017). Fish base. World Wide Web Electronic Publication. (Accessed October $7^{\text {th }}, 2019$ from www.fishbase.org)

Gardner, T. A., Côté, I. M., Gill, J. A., Grant, A., \& Watkinson, A. R. (2003). Long-term region-wide declines in Caribbean corals. Science, 301(5635):958-960.

Giyanto, Manuputty, A., Abrar, M., Siringoringo, R., Suharti, S., Wibowo, K., Edrus, I., Arbi, U., Cappenberg, H., Sihaloho, H., Tuti, Y., \& Zulfianita, D. (2014). Panduan monitoring kesehatan terumbu karang (Issue 1). http://www. coremap.or.id

Graham, N. A. J., Wilson, S. K., Jennings, S., Polunin, N. V. C., Robinson, J., Bijoux, J. P., \& Daw, T. M. (2007). Lag effects in the impacts of mass coral bleaching on coral reef fish, fisheries, and ecosystems. Conservation Biology, 21(5):1291-1300.
Grimsditch, G. D., \& Salm, R. V. (2006). Coral reef resilience and resistance to bleaching. Gland: IUCN.

Gudka, M., Obura, D., Mwaura, J., Porter, S., Yahya, S., \& Mabwa, R. (2018, July). Impact of the 3rd global coral bleaching event on the Western Indian Ocean Impact of the 3rd Global Coral Bleaching Event 633 on the Western Indian Ocean in 2016.

Hammerton, Z. (2017). Determining the variables that influence SCUBA diving impacts in Eastern Australian marine parks. Ocean and Coastal Management, 142:209-217.

Harvell, C. D., Mitchell, C. E., Ward, J. R., Altizer, S., Dobson, A. P., Ostfeld, R. S., \& Samuel, M. D. (2002). Climate warming and disease risks for terrestrial and marine biota. Science, 296(5576):2158-2162.

Harvey, B. J., Nash, K. L., Blanchard, J. L., \& Edwards, D. P. (2018). Ecosystem-based management of coral reefs under climate change. Ecology and Evolution, 8(12):6354-6368.

Haywood, M. D. E., Thomson, D. P., Babcock, R. C., Pillans, R. D., Keesing, J. K., Miller, M., Rochester, W. A., Donovan, A., Evans, R. D., Shedrawi, G., \& Field, S. N. (2019). Crownof-thorns starfish impede the recovery potential of coral reefs following bleaching. Marine Biology, 166(99):1-15.

Head, C. E. I., Bayley, D. T. I., Rowlands, G., Roche, R. C., Tickler, D. M., Rogers, A. D., Koldewey, H., Turner, J. R., \& Andradi-Brown, D. A. (2019). Coral bleaching impacts from backto-back 2015-2016 thermal anomalies in the remote central Indian Ocean. Coral Reefs, 38(4):605-618.

Heery, E. C., Hoeksema, B. W., Browne, N. K., Reimer, J. D., Ang, P. O., Huang, D., Friess, D. A., Chou, L. M., Loke, L. H. L., Saksena-Taylor, P., Alsagoff, N., Yeemin, T., Sutthacheep, M., Vo, S. T., Bos, A. R., Gumanao, G. S., Syed Hussein, M. A., Waheed, Z., Lane, D. J. W., \& Todd, P. A. (2018). Urban coral reefs: Degradation and resilience of hard coral assemblages in coastal cities of East and Southeast Asia. Marine Pollution Bulletin, 135(July), 654-681.

Himes, A. H. (2007). Performance indicator importance in MPA management using a multi-criteria approach. Coastal Management, 35(5):601618. 
Hoegh-Guldberg, O., Poloczanska, E. S., Skirving, W., \& Dove, S. (2017). Coral reef ecosystems under climate change and ocean acidification. Frontiers in Marine Science, 4:1-20.

Hoey, A. S., \& Bellwood, D. R. (2011). Suppression of herbivory by macroalgal density: A critical feedback on coral reefs? Ecology Letters, 14(3):267-273.

Hughes T. P., Anderson, K. D., Connolly, S. R., Heron, S. F., Kerry, J. T., Lough, J. M., Baird, A. H.,Baum, J. K., Berumen, M. L., Bridge, T. C., Claar, D. C., Eakin, C. M., Gilmour, J. P., Graham, N. A. J., Harrison, H., Hobbs, J. P. A., Hoey, A. S., Hoogenboom, M., Lowe, R. J., \& Wilson, S. K. (2018). Spatial and temporal patterns of mass bleaching of corals in the Anthropocene. Science, 359(6371):80-83.

Hughes, T. P, Baird, A. H., Bellwood, D. R., Card, M., Connolly, S. R., Folke, C., \& Grosberg, R. (2003). Climate change, human impacts, and the resilience of coral reefs. Science, 301:929934.

Hughes, T. P., Rodrigues, M. J., Bellwood, D. R., Ceccarelli, D., Hoegh-Guldberg, O., McCook, L., Moltschaniwskyj, N., Pratchett, M. S., Steneck, R. S., \& Willis, B. (2007). Phase shifts, herbivory, and the resilience of coral reefs to climate change. Current Biology, 17(4):360-365.

Jackson, J. B. C., Kirby, M. X., Berger, W. H., Bjorndal, K. A., Botsford, L. W., Bourque, B. J., Bradbury, R. H., Cooke, R., Erlandson, J., Estes, J. A., Hughes, T. P., Kidwell, S., Lange, C. B., Lenihan, H. S., Pandolfi, J. M., Peterson, C. H., Steneck, R. S., Tegner, M. J., \& Warner, R. R. (2001). Historical overfishing and the recent collapse of coastal ecosystems. Science, 293(5530):629-637.

Johnston, M. A., Hickerson, E. L., Nuttall, M. F., Blakeway, R. D., Sterne, T. K., Eckert, R. J., \& Schmahl, G. P. (2019). Coral bleaching and recovery from 2016 to 2017 at East and West Flower Garden Banks, Gulf of Mexico. Coral Reefs, 38(4):787-799.

Jompa, J., \& McCook, L. J. (2003). Contrasting effects of turf algae on corals: Massive Porites spp. are unaffected by mixed-species turfs, but killed by the red alga Anotrichium tenue. Marine Ecology Progress Series, 258:79-86.
Jones, G. P., McCormick, M. I., Srinivasan, M., \& Eagle, J. V. (2004). Coral decline threatens fish biodiversity in marine reserves. Proceedings of the National Academy of Sciences, 101(21):8251-8253.

Kayal, M., Vercelloni, J., Lison de Loma, T., Bosserelle, P., Chancerelle, Y., Geoffroy, S., Stievenart, C., Michonneau, F., Penin, L., Planes, S., \& Adjeroud, M. (2012). Predator crown-ofthorns starfish (Acanthaster planci) outbreak, mass mortality of corals, and cascading effects on reef fish and benthic communities. PLoS ONE, 7(10):1-9.

Keith, S. A., Baird, A. H., Hobbs, J. P. A., Woolsey, E. S., Hoey, A. S., Fadli, N., \& Sanders, N. J. (2018). Synchronous behavioural shifts in reef fishes linked to mass coral bleaching. Nature Climate Change, 8(11):986-991.

Leray, M., Béraud, M., Anker, A., Chancerelle, Y., \& Mills, S. C. (2012). Acanthaster planci outbreak: Decline in coral health, coral size structure modification and consequences for obligate Decapod assemblages. PLOS ONE, 7(4):1-10.

Lin, X., Hu, S., Liu, Y., Zhang, L., Huang, H., \& Liu, S. (2021). Disturbance-mediated changes in coral reef habitat provoke a positive feeding response in a major coral reef detritivore, Ctenochaetus striatus. Frontier of Marine Science, 8:1-14.

Luiz, O. J., Mendes, T. C., Barneche, D. R., Rangel, C. A., Gasparini, L., \& Ferreira, C. E. L. (2015). Community structure of reef fishes on a remote oceanic island (St Peter and $\mathrm{St}$ Paul's Archipelago 704, Equatorial Atlantic): The relative influence of abiotic and biotic variables. Marine and Freshwater Research, 66:739-749.

Luna, B., Pérez, C. V., \& Sánchez-Lizaso, J. L. (2009). Benthic impacts of recreational divers in a Mediterranean marine protected area. ICES Journal of Marine Science, 66(3):517-523.

Madduppa, H. H., Agus, S. B., Farhan, A. R., Suhendra, D., \& Subhan, B. (2014). Fish biodiversity in coral reefs and lagoon at the Maratua Island, East Kalimantan. Biodiversitas, Journal of Biological Diversity, 13(3):145-150.

Marshell, A., \& Mumby, P. J. (2012). Revisiting the functional roles of the surgeon fish Acanthurus nigrofuscus and Ctenochaetus striatus. Coral 
Reefs, 31(4):1093-1101.

McClanahan, T. R., Graham, N. A. J., MacNeil, M. A., Muthiga, N. A., Cinner, J. E., Bruggemann, J. H., \& Wilson, S. K. (2011). Critical thresholds and tangible targets for ecosystembased management of coral reef fisheries. Proceedings of the National Academy of Sciences, 108(41):17230-17233.

McClanahan, T. R., \& Jadot, C. (2017). Managing coral reef fish community biomass is a priority for biodiversity conservation in Madagascar. Marine Ecology Progress Series, 580:169190.

McCulloch, M., Fallon, S., Wyndham, T., Hendy, E., Lough, J., \& Barnes, D. (2003). Coral record of increased sediment flux to the inner Great Barrier Reef since European settlement. Nature, 421(6924):727-730.

McMahon, A., Santos, I. R., Schulz, K. G., Scott, A., Silverman, J., Davis, K. L., \& Maher, D. T. (2019). Coral reef calcification and production after the 2016 bleaching event at Lizard Island, Great Barrier Reef. Journal of Geophysical Research: Oceans, 124(6):4003-4016.

McManus, J. W., \& Polsenberg, J. F. (2004). Coralalgal phase shifts on coral reefs: Ecological and environmental aspects. Progress in Oceanography, 60(2-4):263-279.

Mellin, C., Macneil, A. M., Cheal, A. J., Emslie, M. J., \& Caley, J. M. (2016). Marine protected areas increase resilience among coral reef communities. Ecology Letters, 19(6):629-637.

Meyer, A. L., \& Dierking, J. (2011). Elevated size and body condition and altered feeding ecology of the grouper Cephalopholis argus in nonnative habitats. Marine Ecology Progress Series, 439(737):202-212.

Moberg, F., \& Folke, C. (1999). Coral reefs goods and services. Ecological Economics, 29:215-233.

Morgan, K. M., Perry, C. T., Johnson, J. A., \& Smithers, S. G. (2017). Nearshore turbid-zone corals exhibit high bleaching tolerance on the Great Barrier Reef following the 2016 ocean warming event. Frontiers in Marine Science, $4: 1-13$.

Mumby, P. J., Dahlgren, C. P., Harborne, A. R., Kappel, C. V., Micheli, F., Brumbaugh, D. R., Holmes, K. E., Mendes, J. M., Broad, K., Sanchirico, J. N., Buch, K., Box, S., Stoffle, R. W., \& Gill,
A. B. (2006). Fishing, trophic cascades, and the process of grazing on coral reefs. Science, 311(5757):98-101.

Mumby, P. J., Harborne, A. R., Williams, J., Kappel, C. V., Brumbaugh, D. R., Micheli, F., Holmes, K. E., Dahlgren, C. P., Paris, C. B., \& Blackwell, P. G. (2007). Trophic cascade facilitates coral recruitment in a marine reserve. Proceedings of the National Academy of Sciences of the United States of America, 104(20):8362-8367.

Newman, M. J. H., Paredes, G. A., Sala, E., \& Jackson, J. B. C. (2006). Structure of Caribbean coral reef communities across a large gradient of fish biomass. Ecology Letters, 9(11):12161227.

Nishiguchi, S., Wada, N., Yamashiro, H., Ishibashi, H., \& Takeuchi, I. (2018). Continuous recordings of the coral bleaching process on Sesoko Island, Okinawa, Japan, over about 50 days using an underwater camera equipped with a lens wiper. Marine Pollution Bulletin, 131:422-427.

Nizam, I., Mohamed, M., Basheer, A., Haleem, I., Nistharan, F., Schmidt, A., Naeem, R., Abdulla, A., \& Grimsditch, G. (2016). Status of coral bleaching in the Maldives 2016. Maldives: Marine Research Center.

Paddack, M. J., Reynolds, J. D., Aguilar, C., Appeldoorn, R. S., Beets, J., Burkett, E. W., Chittaro, P. M., Clarke, K., Esteves, R., Fonseca, A. C., Forrester, G. E., Friedlander, A. M., García-Sais, J., González-Sansón, G., Jordan, L. K. B., McClellan, D. B., Miller, M. W., Molloy, P. P., Mumby, P.J., \& Côté, I. M. (2009). Recent region-wide declines in $\mathrm{Ca}$ ribbean reef fish abundance. Current Biology, 19(7):590-595.

Pandolfi, J. M., Bradbury, R. H., Sala, E., Hughes, T. P., Bjorndal, K. A., Cooke, R. G., McArdle, D., McClenachan, L., Newman, M. J. H., Paredes, G., Warner, R. R., \& Jackson, J. B. C. (2003). Global trajectories of the long-term decline of coral reef ecosystems. Science, 301(5635):955-958.

Pinheiro, H. T., Ferreira, C. E. L. L., Joyeux, J.-C. C., Santos, R. G., \& Horta, P. A. (2011). Reef fish structure and distribution in a South-Western Atlantic Ocean tropical island. Journal of Fish Biology, 79(7):1984-2006.

Pinheiro, H. T., Madureira, C. J. M., Joyeux, J. C., \& Martins, A. S. (2015). Fish diversity of a 
South-Western Atlantic coastal island: Aspects of distribution and conservation in a marine zoogeographical boundary. Check List, 11(2):1615.

Pratchett, M. S., Wilson, S. K., \& Baird, A. H. (2006). Declines in the abundance of Chaetodon butterfly fishes following extensive coral depletion. Journal of Fish Biology, 69(5):12691280 .

Pratchett, Morgan S., Caballes, C. F., Wilmes, J. C., Matthews, S., Mellin, C., Sweatman, H. P. A., Nadler, L. E., Brodie, J., Thompson, C. A., Hoey, J., Bos, A. R., Byrne, M., Messmer, V., Fortunato, S. A. V., Chen, C. C. M., Buck, A. C. E., Babcock, R. C., \& Uthicke, S. (2017). Thirty years of research on crown-of-thorns starfish (1986-2016): Scientific advances and emerging opportunities. Diversity, 9(4):41.

Pratchett, M. S., Hoey, A. S., Wilson, S. K., Messmer, V., \& Graham, N. A. J. (2011). Changes in biodiversity and functioning of reef fish assemblages following coral bleaching and coral loss. Diversity, 3(3):424-452.

Putra, R. D., Suhana, M. P., Kurniawn, D., Abrar, M., Siringoringo, R. M., Sari, N. W. P., Irawan, H., Prayetno, E., Apriadi, T., \& Suryanti, A. (2019, April). Detection of reef scale thermal stress with aqua and terra MODIS satellite for coral bleaching phenomena. AIP Conference Proceedings, 2094(1):020024.

Putra, R. D., Suryanti, A., Kurniawan, D., Pratomo, A., Irawan, H., Said Raja'I, T., Kurniawan, R., Pratama, G., \& Jumsurizal. (2018). Responses of herbivorous fishes on coral reef cover in outer island Indonesia (Study Case: Natuna Island). E3S Web of Conferences, 47(04009):1-18.

Ranith, R., \& Kripa, V. (2019). Post-bleaching benthic status on reef sites of Mandapam group of Islands and Palk Bay, Indian Ocean following the 2016 bleaching events. Regional Studies in Marine Science, 27(8):100525.

Raymundo, L. J., Burdick, D., Hoot, W. C., Miller, R. M., Brown, V., Reynolds, T., Gault, J., Idechong, J., Fifer, J., \& Williams, A. (2019). Successive bleaching events cause mass coral mortality in Guam, Micronesia. Coral Reefs, 38(4):677-700.

Robinson, J. P. W., Wilson, S. K., Jennings, S., \& Graham, N. A. J. (2019). Thermal stress induces persistently altered coral reef fish assemblages. Global Change Biology, 25(8):2739-2750.

Rouphael, A. B., \& Inglis, G. J. (1997). Impacts of recreational SCUBA diving at sites with different reef topographies. Biological Conservation, 82(3):329-336.

Rudi, E., Elrahimi, S. A., Kartawijaya, T., Herdiana, Y., Setiawan, F., Pardede, S. T., Campbell, S. J., \& Tamelander, J. (2014). Reef fish status in Northern Acehnese reef based on management type. Biodiversitas, Journal of Biological Diversity, 10(2):88-93.

Sandin, S. A., Sampayo, E. M., \& Vermeij, M. J. A. (2008a). Coral reef fish and benthic community structure of Bonaire and Curaçao, Netherlands Antilles. Caribbean Journal of Science, 44(2):137-144.

Sandin, S. A., Smith, J. E., DeMartini, E. E., Dinsdale, E. A., Donner, S. D., Friedlander, A. M.,Konotchick, T., Malay, M., Maragos, J. E., Obura, D., Pantos, O., Paulay, G., Richie, M., Rohwer, F., Schroeder, R. E., Walsh, S., Jackson, J. B. C., Knowlton, N., \& Sala, E. (2008b). Baselines and degradation of coral reefs in the Northern Line Islands. PLoS ONE, 3(2):1-11.

Swierts, T., \& Vermeij, M. J. A. (2016). Competitive interactions between corals and turf algae depend on coral colony form. PeerJ, 2016(5):1-18.

Tebbett, S. B., Goatley, C. H. R., \& Bellwood, D. R. (2017). Clarifying functional roles: algal removal by the surgeon fishes Ctenochaetus striatus and Acanthurus nigrofuscus. Coral Reefs, 36(3):803-813.

Tebbett, S. B., Goatley, C. H. R., Huertas, V., Mihalitsis, M., \& Bellwood, D. R. (2018). A functional evaluation of feeding in the surgeon fish Ctenochaetus striatus: The role of soft tissues. Royal Society Open Science, 5(1):1-10.

Tebbett, S. B., Streit, R. P., \& Bellwood, D. R. (2019). Expansion of a colonial Ascidian following consecutive mass coral bleaching at Lizard Island, Australia. Marine Environmental Research, 144:125-129.

Teixeira, C. D., Leitão, R. L. L., Ribeiro, F. V., Moraes, F. C., Neves, L. M., Bastos, A. C., Pereira-Filho, G. H., Kampel, M., Salomon, P. S., Sá, J. A., Falsarella, L. N., Amario, M., Abieri, M. L., Pereira,R. C., Amad o-Filho, G. M., \& Moura, 
R. L. (2019). Sustained mass coral bleaching (2016-2017) in Brazilian turbid-zone reefs: taxonomic, cross-shelf and habitat-related trends. Coral Reefs, 38(4):801-813.

Thibaut, L. M., \& Connolly, S. R. (2013). Understanding diversity-stability relationships: Towards a unified model of portfolio effects. Ecology Letters, 16(2):140-150.

Thinesh, T., Meenatchi, R., Jose, P. A., Kiran, G. S., \& Selvin, J. (2019). Differential bleaching and recovery pattern of southeast Indian coral reef to 2016 global mass bleaching event: Occurrence of stress-tolerant symbiont Durusdinium (Clade D) in corals of Palk Bay. Marine Pollution Bulletin, 145:287-294.

Titlyanov, E. A., Yakovleva, I. M., \& Titlyanova, T. V. (2007). Interaction between benthic algae (Lyngbya bouillonii, Dictyota dichotoma) and scleractinian coral Porites lutea in direct contact. Journal of Experimental Marine Biology and Ecology, 342(2):282-291.

Tratalos, J. A., \& Austin, T. J. (2001). Impacts of recreational SCUBA diving on coral communities of the Caribbean island of Grand Cayman. Biological Conservation, 102(1):6775.

Trenouth, A., \& Campbell, M. (2013). Perceptions of ecological risk associated with introduced marine species in marine protected areas. Management of Biological Invasions, 4(1):714.

Tricas, T. (1989). Determinants of feeding territory size in the coralivorous butterflyfish. Animal Behaviour, 37:830-841.

Vergés, A., Bennett, S., \& Bellwood, D. R. (2012). Diversity among macroalgae-consuming fishes on coral reefs: A transcontinental comparison. PLoS ONE, 7(9):e45543.

West, J. M., \& Salm, R. V. (2003). Resistance and resilience to coral bleaching: Implications for coral reef conservation and management. Conservation Biology, 17(4):956-967.

Wild, C., Jantzen, C., \& Kremb, S. G. (2014). Turf algae-mediated coral damage in coastal reefs of Belize, Central America. PeerJ, 2014(1):1-14.

Wilkinson, C. (2008). Status of coral reefs of the world: 2008. Townsville: Global Coral Reef Monitoring Network.
Wilson, S. K., Graham, N. A. J., Fisher, R., Robinson, J., Nash, K., Chong-Seng, K., Polunin, N. V. C., Aumeeruddy, R., \& Quatre, R. (2012). Effect of macroalgal expansion and marine protected areas on coral recovery following a climatic disturbance. Conservation Biology, 26(6):995-1004.

Wismer, S., Tebbett, S. B., Streit, R. P., \& Bellwood, D. R. (2019). Spatial mismatch in fish and coral loss following 2016 mass coral bleaching. Science of the Total Environment, 650:14871498.

Wolanski, E., Andutta, F., Deleersnijder, E., Li, Y., \& Thomas, C. J. (2017). The Gulf of Carpentaria heated Torres Strait and the Northern Great Barrier Reef during the 2016 mass coral bleaching event. Estuarine, Coastal and Shelf Science, 194:172-181.

Woodhead A. J., Hicks, C. C., Norström A. V, Williams, N A., \& Graham, N A. J. (2019) Coral reef ecosystem services in the Anthropocene. Functional Ecology, 33(6):1023-1034.

Wouthuyzen, S., Abrar, M., \& Lorwens, J. (2018). A comparison between the 2010 and 2016 ElNinō induced coral bleaching in the Indonesian waters. IOP Conference Series: Earth and Environmental Science, 118:012051.

Wouthuyzen, S., Abrar, M., Corvianawatie, C., Kusumo, S., Yanuar, Y, Dermawan, D., Yennafri, Y., Salatalohi, A., Hanif, A., Permana, S., \& Arafat, M., Y. (2020). Trend of sea surface temperature increase and coral resilience after bleaching events of 2010 and 2016 in The Marine Tourism Park (TWP) of Pieh Island, Padang, West Sumatra. Oseanologi dan Limnologi di Indonesia, 5(1):1-18.

Wu, S. T. (2014). Marine recreational activity impact on Kengting National Park, Taiwan. Journal of Hospitality Management and Tourism, 4(1):18.

Yahya, S. A. S., Gullström, M., Öhman, M. C., Jiddawi, N. S., Andersson, M. H., Mgaya, Y. D., \& Lindahl, U. (2011). Coral bleaching and habitat effects on colonisation of reef fish assemblages: An experimental study. Estuarine, Coastal and Shelf Science, 94(1):16-23.

Zenone, A. M., Burkepile, D. E., \& Boswell, K. M. (2017). A comparison of diver vs. acoustic methodologies for surveying fishes in a shallow water coral reef ecosystem. Fisheries Research, 189:62-66. 